\title{
Adsorption of metal nanoparticles on carbon substrates and epitaxial graphene: Assessing models for dispersion forces
}

\author{
G. D. Förster and F. Rabilloud \\ ILM, Université Lyon 1-CNRS UMR5306, Université de Lyon, 10 Rue Ada Byron, 69622 Villeurbanne Cedex, France \\ F. Calvo* \\ LIPhy, Univ. Grenoble 1 and CNRS, UMR5588, 140, Av. de la physique, 38402 St Martin D'Hères, France
}

(Received 17 April 2015; revised manuscript received 5 June 2015; published 29 June 2015)

\begin{abstract}
Carbon substrates such as graphite or epitaxial graphene can be employed to support metal nanoparticles for applications in diverse areas of surface science. In this paper, we address the computational modeling of such systems by means of semiempirical potentials, and in particular the possible role of long-range London dispersion forces. Following the Grimme (D2) strategy often used in combination with density-functional theory calculations, we propose some analytical extensions taking into account the crystalline and semi-infinite natures of the substrate and, in the case of epitaxial graphene, the possible screening of the van der Waals interaction by the bulk underlying metal. These ideas are tested in the specific case of platinum nanoparticles deposited on graphene, graphite, and graphene epitaxially grown on $\mathrm{Pt}(111)$ modeled using a many-body Brenner-type potential, and validated against available electronic-structure calculations. Systematic optimizations carried out at zero temperature indicate the relative stability of various nanoparticle shapes on their support, for adsorbates containing several thousand atoms. Using molecular dynamics simulations, we shed light on the thermal behavior and emphasize the key role of dispersion forces on the stabilization of the adsorbates at finite temperature. The vibrational properties of graphene layers in contact with a Pt nanoparticle or epitaxially grown on $\mathrm{Pt}(111)$ also reveal some clear sensitivity on temperature and strain.
\end{abstract}

DOI: 10.1103/PhysRevB.91.245433

PACS number(s): 82.60.Qr, 81.05.ue, 81.05.uf, 61.43.Bn

\section{INTRODUCTION}

The use of nanoparticles (NPs) for practical applications requires stabilizing them against thermal motion and protecting them against possible degradation and reactions due to the external medium, e.g., through appropriate coating with surfactant molecules. In surface science, the particles are either grown in situ from the atoms or deposited after their initial formation in the gas phase [1-3]. Owing to their high versatility, relative chemical inertness, and reasonable production costs, carbon materials are often employed as substrates for NP deposition [4-10].

Platinum nanoparticles interacting with carbon substrates have become an active field of research due to numerous potential applications in catalysis [8-11] or in hydrogen-based technologies [12]. One motivation is clearly to exploit the high surface/volume of nanoparticles in order to reduce the amount needed of this expensive metal. Platinum NPs have thus been used in fuel cells as catalysts for oxygen reduction but also for promoting hydrogen oxidation at low temperature $[8,9,13]$. Pt nanoparticles have also been shown to enhance the hydrogen storage capacity of carbon porous nanomaterials such as zeolites [12]. By favoring dissociative chemisorption of hydrogen at their surface, nanoparticles contribute to diffuse hydrogen toward adjacent surfaces, a process commonly referred to as hydrogen spillover. The selective permeability of graphene toward thermal protons but not to molecular hydrogen has recently been found to be further increased by platinum adsorbates [14]. All these applications need well-distributed and stable assemblies of Pt clusters, which in

*florent.calvo@ujf-grenoble.fr turn requires that the mechanisms of formation and adsorption of such nanomaterials are fully understood.

One important contribution to the interaction of adsorbates with bulk or semi-infinite substrates is that of long-range dispersion forces [15-24]. Although conceptually London dispersion interactions are already treated naturally by firstprinciples methods such as density-functional theory (DFT), long-range electron correlations are not taken into account within local and semilocal exchange correlation functionals. This issue is particularly critical in the case of adsorbates interacting weakly with their substrate via physisorption, for which dispersion is the dominant binding force. To overcome this shortcoming, several dedicated approaches have recently been developed [25], relying either on electronic densitybased corrections or on empirical pairwise approximations. The former class of methods includes both truly nonlocal functionals [26,27] and the highly parametrized forms of metahybrid approximations [28,29]. The second class of methods, which includes DFT-D, uses in its most basic form a pairwise atomistic additive correction of dispersion with a $C_{6}$ coefficient and a damping function [30,31] for which several parametrizations and expressions have been proposed [32-36].

Among the conclusions of those recent developments, dispersion forces were found to be significantly influenced by the local environment, appropriate $C_{6}$ coefficients possibly varying by one order of magnitude depending on the size and shape of the structure $[21,37,38]$. If the substrate is metallic or semiconducting, electron delocalization can also screen dispersion forces, which introduces some dependence on temperature and on the range, notably through retardation effects at longmost distances [39]. Such effects have been investigated also experimentally in the case of organic molecules in contact with a metal surface [40]. 
Dispersion forces are of course essential in describing the adsorption of molecules on extended substrates, not only in the context of physisorption for which they are the cause, but also in their alteration of chemisorption interactions. The magnitude of long-range van der Waals forces has notably been quantified in an increasing number of electronic-structure investigations of complex substrates [15,16,41], individual adsorbates [15], or molecular self-assemblies [24,42]. In the case of small Pt clusters adsorbed on graphite, recent DFT calculations have confirmed their significance on the binding energy [43] in the static, 0-K limit. While the DFT approach currently stands as the most realistic way of treating atomic and molecular interactions in complex systems, it is computationally limited in terms of size and time scales. The alternative description by semiempirical analytical potentials does not suffer from such limitations, but of course to the price of chemical accuracy. Interestingly enough, force fields to model covalent or metallic materials also typically neglect dispersion forces altogether rather similarly to the way DFT approaches were neglecting those corrections until the last decade (and still often do). Dispersion interactions between metal adsorbates and extended substrates have been explicitly taken into account in some empirical models based on the Lennard-Jones (LJ) potential [44] but otherwise lacking chemical consistency.

The description of physisorption phenomena on semiinfinite substrates by analytical potentials has been conveniently described by Steele [45] who integrated the LennardJones potential over a succession of crystallographic planes. In this paper, we propose an extension of this approach to the case of platinum adsorbates on carbon substrates, taking into account the possibility of chemisorption as well. Our strategy is based on a Brenner-type potential [46] that is able to model both covalent and metallic parts of the system, and also mixtures of the two elements after appropriate parametrization [47]. We have enriched this potential with dispersion corrections in order to gain the ability of modeling correctly physisorption phenomena that are potentially essential for nanoparticles in contact with extended environments. Those corrections follow the Grimme D2 model [31] employed for DFT calculations, as well as the Steele approach for integrating the van der Waals energy over the entire support. The Brenner force field has been further extended to include noncovalent interactions in the case of electronically delocalized substrates, as present, e.g., in epitaxial graphene on metals.

The purpose of this work is to critically discuss the importance of dispersion interactions on the physical behavior of platinum nanoparticles on a variety of ordered carbon substrates ranging from graphene to multilayer graphite and epitaxial graphene on $\operatorname{Pt}(111)$. The various coarse-grained models we are proposing here are adapted to these different situations, and compared against all-atom calculations at zero and finite temperature on various observables such as adsorption energies, dynamical indicators, or vibrational spectra. One main conclusion is that dispersion forces contribute significantly to the adsorption energy and that the uppermost layers of the substrate dominate this contribution.

In the following section, we present the atomistic potential which additively combines covalent and dispersion interactions, and we derive a coarse-grained implicit model in the case of adsorbates deposited on semi-infinite carbonated substrates. Different variants of the dispersion model are compared on simple test cases and for more realistic adsorbates on graphite at finite temperature in Sec. III. The model is further extended in Sec. IV to describe the specific interactions of epitaxial graphene on $\mathrm{Pt}(111)$ possibly acting as a substrate for Pt nanoparticles, and again at zero and finite temperatures. Our results here emphasize the importance of screening and nonadditivity of the van der Waals interactions on the resulting dynamical behavior. Finally, in Sec. V we summarize and suggest other applications of the present methodology to related systems.

\section{BOND-ORDER POTENTIAL AND DISPERSION CORRECTIONS}

Since their introduction for covalent systems $[48,49]$ and their unification [50] with embedded-atom models [51,52], bond-order potentials have been routinely used to model a broad variety of materials. Their ability to describe at once metallic and covalent systems in contact has motivated us to choose such a potential to model platinum nanoparticles on carbon substrates. However, in their original form such potentials lack long-range dispersion forces and it is the purpose of this section to make this extension, especially in the case of semi-infinite substrates.

The generic system we consider is one metallic adsorbates or nanoparticles deposited on purely graphitic substrates ranging from a graphene monolayer to multilayer graphite, or on graphene epitaxially grown on the same metal.

\section{A. Covalent and metallic contributions}

We first assume that the entire system is explicitly described as a set of atoms located at the collective position $\mathbf{R}=\left\{\vec{r}_{i}\right\}$. The covalent and metallic parts of the total binding energy are expressed under the following form $[46,49]$ :

$$
E=\sum_{i} \sum_{j<i} f^{i j}\left(r_{i j}\right)\left(V_{R}-\bar{b}_{i j} V_{B}\right)
$$

with $r_{i j}$ the distance between atoms $i$ and $j, V_{R}$ and $V_{B}$ two exponential functions corresponding to the repulsive and binding contributions

$$
\begin{aligned}
& V_{R}=\frac{D_{0}^{i j}}{S^{i j}-1} e^{-\beta^{i j} \sqrt{2 S^{i j}\left(r_{i j}-r_{0}^{i j}\right)}}, \\
& V_{B}=\frac{S^{i j} D_{0}^{i j}}{S^{i j}-1} e^{-\beta^{i j} \sqrt{2 / S^{i j}\left(r_{i j}-r_{0}^{i j}\right)}},
\end{aligned}
$$

$D_{0}^{i j}, S^{i j}, \beta^{i j}$, and $r_{0}^{i j}$ being four sets of three parameters that depend on the nature of the two elements involved. In Eq. (1), $\bar{b}_{i j}$ is the bond order associated with pair $(i, j)$ of atoms:

$$
\begin{gathered}
\bar{b}_{i j}=\frac{1}{2}\left(b_{i j}+b_{j i}\right), \\
b_{i j}=\left\{1+\sum_{k \neq i, j} f^{i k}\left(r_{i k}\right) g(\Theta) e^{2 \mu_{i j k}^{\left[\left(r_{i j}-r_{0}^{i j}\right)-\left(r_{i k}-r_{0}^{i k}\right)\right]}}\right\}^{-\frac{1}{2}},
\end{gathered}
$$


where the additional $\pi$ conjugation terms required to better describe hydrocarbon molecules [46] were not taken into account here [47].

In this expression, $\Theta=\widehat{j i k}$ is the bending angle between atoms $i, j$, and $k$ and $g(\Theta)$ has the explicit form

$$
g(\Theta)=\gamma_{i j k}\left[1+\left(\frac{c_{i j k}^{2}}{d_{i j k}^{2}}-\frac{c_{i j k}^{2}}{d_{i j k}^{2}+(1+\cos \Theta)^{2}}\right)\right],
$$

where $\mu_{i j k}, \gamma_{i j k}, c_{i j k}$, and $d_{i j k}$ are additional parameters. Finally, a pairwise cutoff function $f^{i j}$ is introduced so interactions are limited to the range of chemical bonding:

$$
f^{i j}(r)=\left\{\begin{array}{lc}
1, & r<R_{i j}^{(1)} \\
\frac{1}{2}\left[1+\cos \left(\pi \frac{r-R_{i j}^{(1)}}{R_{i j}^{(2)}-R_{i j}^{(1)}}\right)\right], & R_{i j}^{(1)} \leqslant r \leqslant R_{i j}^{(2)} \\
0, & r>R_{i j}^{(2)}
\end{array}\right.
$$

with $R_{i j}^{(1)}$ and $R_{i j}^{(2)}$ the inner and outer cutoff radii. The various parameters of the potential have been specifically optimized for the Pt-C system by Albe and co-workers [47], and we use those parameters here without any modification.

\section{B. Explicit Grimme-type dispersion models}

Long-range dispersion forces are missing from the previous Pt-C bond-order potential, and as such the model cannot properly describe physisorption phenomena that can be important especially for adsorbates on semi-infinite substrates. The approach we follow to solve this shortcoming is similar to the Grimme approach in DFT [31], and consists of adding a posteriori some dispersion energy to the covalent, metallic, and ionic contributions. In the fully atomistic Grimme D2 approach [31], the dispersion correction reads as

$$
E_{\mathrm{disp}}^{\mathrm{D} 2}=-s_{6} \sum_{i} \sum_{j \neq i} \frac{C_{6}^{i j}}{r_{i j}^{6}} f_{\mathrm{dmp}}^{i j}\left(r_{i j}\right),
$$

where $C_{6}^{i j}=\left(C_{6}^{i} C_{6}^{j}\right)^{1 / 2}$ are the parameters of the dominant van der Waals (vdW) contributions, $f_{\mathrm{dmp}}^{i j}$ being a Fermi-type damping function so that dispersion only acts at long range and does not alter the covalent part:

$$
f_{\mathrm{dmp}}^{i j}\left(r_{i j}\right)=\frac{1}{1+\exp \left[-20\left(\frac{r_{i j}}{R_{r}^{i j}}-1\right)\right]},
$$

$R_{r}^{i j}$ being the sum of van der Waals atomic radii $R_{r}^{i}$. In Eq. (6), $s_{6}$ is a prefactor to be adjusted depending on the functional. We adopt here the value $s_{6}=1.05$ as indicated for the purely dispersionless functional BP86 [31].

For the present problem of Pt nanoparticles on carbonated substrates, the values of the other coefficients were taken straight from the Grimme source code [31] and read as $C_{6}^{\mathrm{C}}=18.1376 \mathrm{eV}^{6}, C_{6}^{\mathrm{Pt}}=842.0014 \mathrm{eV}^{6}, R_{r}^{\mathrm{C}}=1.452 \AA$, and $R_{r}^{\mathrm{Pt}}=1.7721 \AA$. Finally, and in contrast to Grimme's original formulation, we consider dispersion interactions only up to the size of the simulation box, neglecting interactions of the system with itself.

We have also tried the more recent and more realistic D3 version of Grimme's dispersion correction scheme [32,33], in which the short-range damping takes another form and additional $C_{8}$ terms are considered and the van der Waals coefficients are given further flexibility with some dependence on the local coordination number $(\mathrm{CN})$ of individual atoms, taken as in Refs. [32,33] as an interpolation between tabulated values at discrete numbers and using the same functional form as in those references. The coordination number of atom $i$ in the D3 model is expressed as [32]

$$
\mathrm{CN}^{i}=\sum_{j \neq i} \frac{1}{1+\exp \left[-16\left(\frac{4}{3} \frac{R_{\mathrm{cov}}^{i}+R_{\mathrm{cov}}^{j}}{r_{i j}}-1\right)\right]}
$$

with $R_{\text {cov }}$ the covalent radius of the corresponding atom [53]. The parameters for the D3 dispersion model were taken from Refs. [32,33], the covalent radii from Ref. [53]. However, this added complexity produces a much less favorable scaling with increasing number of atoms [in $\mathcal{O}\left(n^{3}\right)$ ], even compromising the use of the bond-order potential in the first place. The D3 dispersion model was thus used occasionally for comparison with the explicit D2 model and only for static properties, with explicit dispersion interactions being evaluated only up to the first periodic images of the system.

\section{Implicit dispersion corrections for adsorption on pure carbonated substrates}

Next, we consider the semi-infinite extension of the carbon substrate, assuming for notations that the surface of the substrate lies perpendicular to the $z$ axis. When using periodic boundary conditions, only the immediate contributions to the dispersion energy are explicitly included, and it may be necessary to integrate the missing contribution away from the primitive cell. These ideas were originally developed by Steele [45] who integrated the Lennard-Jones potential for adsorption over an infinite stack of flat crystalline layers. One additional subtlety here is the presence of a short-range cutoff in the dispersion correction, which must be taken into account when carrying out the integration.

Aiming for a similar coarse-grained (implicit) model of the adsorbate-substrate dispersion interaction, we thus assume that the support can be described by a succession of flat layers with surface density $\sigma$. In the case of epitaxial graphene, $\sigma$ will denote the density of the topmost graphene layer, all $\operatorname{Pt}(111)$ layers underneath having a possibly different surface density $\sigma^{\prime}$. Although the following coarse-graining procedure can be used for integrating the many-body (but short-ranged) component of the interactions [54], we use it here only for the long-range dispersion forces that are more sensitive to the infinite nature of the environment.

The interaction of the adsorbate with the first layer can be integrated as follows. We denote by $d_{i}=z_{i}-\bar{z}$ the separation along the $z$ axis of atom $i$ of the adsorbate to the average layer position $\bar{z}$. For integration purposes, we temporarily replace the Fermi-type damping function of the Grimme D2 model by a sharp short-distance cutoff placed where the original $f_{\mathrm{dmp}}^{i j}$ equals $\frac{1}{2}$, namely, at the position $r_{i j}=R_{r}^{i j}$ :

$$
f_{\mathrm{dmp}}^{i j}\left(r_{i j}\right) \approx \begin{cases}0, & r_{i j} \leqslant R_{r}^{i j} \\ 1, & \text { otherwise }\end{cases}
$$


The dispersion interaction of an adsorbate (ads) with a single substrate layer is then integrated as

$$
\widetilde{E}_{\mathrm{disp}}^{(1)}=\frac{-s_{6} \sigma C_{6} \pi}{2} \sum_{i \in \mathrm{ads}} \begin{cases}R_{r}^{-4}, & \left|d_{i}\right|<R_{r} \\ d_{i}^{-4}, & \text { otherwise }\end{cases}
$$

where $C_{6}$ is chosen appropriately to the substrate atom under consideration (Pt-C) and $R_{r}$ denotes the cutoff of the dispersion interaction also chosen for Pt-C.

Due to the introduction of a sharp cutoff in $f_{\mathrm{dmp}}$, this expression is not differentiable at $d_{i}=R_{r}$, hence, we replace the constant part by an expression of the form $a d_{i}^{n}+b$, set to match the original expression at $d_{i}=0$ and to produce a continuously differentiable function:

$$
\widetilde{E}_{\text {disp }}^{(1)}=\frac{-s_{6} \sigma C_{6} \pi}{2} \sum_{i \in \text { ads }} \begin{cases}-\frac{4}{n}\left[\left(1+\frac{4}{n}\right)^{\frac{1}{4}} R_{r}\right]^{-4-n} d_{i}^{n}+R_{r}^{-4}, & \left|d_{i}\right|<R_{r}\left(1+\frac{4}{n}\right)^{\frac{1}{4}} \\ d_{i}^{-4}, & \text { otherwise. }\end{cases}
$$

For $n \rightarrow \infty$, the original form is recovered. Choosing $n=50$ is a good compromise between an acceptable approximation and a sufficiently low curvature.

Equation (11) is appropriate for describing the van der Waals contribution from the single graphene monolayer, but requires further summation for graphite. We thus sum the dispersion energy between the adsorbate and the infinite stack of layers of the substrate that we assume are distant from one another by a fixed quantity $d_{L}$. In summing up the above expression of Eq. (11), the condition $\left|d_{i}\right|<R_{r}(1+4 / n)^{1 / 4}$ is satisfied only up to a finite number of uppermost layers, as depicted in Fig. 1. In practice, this concerns the first two layers, for which Eq. (11) has to be explicitly used. For the remaining layers, the summation leads to

$$
\begin{aligned}
\widetilde{E}_{\mathrm{disp}}^{(\infty)} & =-\frac{s_{6} \sigma C_{6} \pi}{2} \sum_{i \in \text { ads }} \sum_{j=0}^{\infty}\left(j d_{L}+d_{i 3}\right)^{-4} \\
& =-\frac{s_{6} \sigma C_{6} \pi}{2 d_{L}^{4}} \sum_{i \in \text { ads }} \zeta\left(4, \frac{d_{i 3}}{d_{L}}\right)
\end{aligned}
$$

where $d_{i 3}=z_{i}-\bar{z}+d_{L}^{1}+d_{L}$ is the distance between an adsorbate atom and the third substrate layer and $d_{L}^{1}$ the spacing between the first two substrate layers. For graphite, $d_{L}^{1}=d_{L}$. In the previous equation, $\zeta$ denotes the Hurwitz zeta

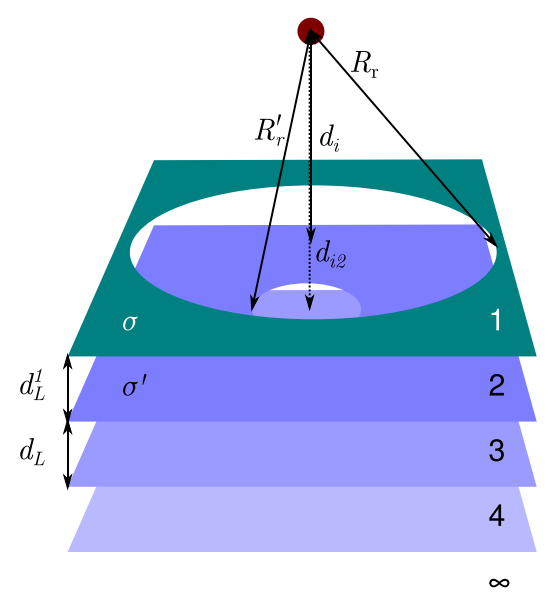

FIG. 1. (Color online) Interaction between an adsorbate atom $i$ and a succession of planar layers, smoothly excluding regions within cutoff distances. See text for notations. function, which in practice was numerically evaluated using the gsl_sf_hzeta function of the GNU Scientific Library.

In the case of the substrates considered here, the first layer is always a graphene sheet with an atomic surface density of $\sigma=4 /\left(\sqrt{3} a^{2}\right)$ of carbon atoms, where $a=2.51 \AA$ is the graphene lattice parameter at $0 \mathrm{~K}$ (as computed with the present Brenner potential). The same surface density is used for all other layers in the case of the graphite substrate. For epitaxial graphene on fcc platinum oriented on the (111) surface and in a $(9 \times 9) \mathrm{C} /(8 \times 8) \mathrm{Pt}$ arrangement, the platinum layers all have the surface density $\sigma_{m}=2 /\left(\sqrt{3} a_{m}^{2}\right)$ with $a_{m}=2.83 \AA$ the first-neighbor distance of Pt atoms in the fcc lattice.

As a final comment for this section, it should be emphasized that the coarse-grained model for dispersion corrections lacks lateral corrugations, in contrast with the original Steele potentials where binding is purely of the van der Waals type [45]. In the present models, lateral corrugation over the carbonated substrates is included through the bond-order contribution, which is always explicit. Lateral corrugation due to the periodic nature of the crystalline substrate is assumed to be much lower than the integrated contribution from the semi-infinite medium, and is therefore neglected.

\section{Intragraphite dispersion interactions}

The previously described coarse-grained implicit model for dispersion interactions has to be benchmarked against fully atomistic treatments. However, the original Brenner potential is not able to bind graphite layers together, and adding dispersion corrections would not suffice because the covalent part does not operate at the experimental interlayer distance due to a too short cutoff. It is thus necessary to also modify the repulsive component of the potential, and following Che and co-workers [55] we address these two issues simply by adding a Lennard-Jones potential between atoms of different layers,

$$
E_{i}^{\mathrm{LJ}}=\sum_{j} \frac{\varepsilon}{2}\left[\left(\frac{\xi}{r_{i j}}\right)^{12}-2\left(\frac{\xi}{r_{i j}}\right)^{6}\right]
$$

where the primed sum indicates that the $j$ atoms belong to a different layer than $i$. For this contribution, we adopt the same parameters as in Ref. [55], namely, $\varepsilon=3 \mathrm{meV}$ and $\xi=3.805 \AA$. The LJ interaction is included in explicit models of graphite. 
In order to derive a fully implicit model, we integrate Eq. (14) above over the flat monolayer to get the well-known result [45]

$$
E_{\mathrm{LJ}, \mathrm{i}}^{(1)}=\frac{\pi \sigma \varepsilon}{2}\left(\frac{\xi^{12}}{5 d_{i}^{10}}-\frac{\xi^{6}}{d_{i}^{4}}\right)
$$

in which $d_{i}$ is the average distance between atom $i$ and the layer. $E_{\mathrm{LJ}}^{(1)}$ is minimal for $d_{i}=d_{\mathrm{C}}^{\min }=2^{-1 / 6} \xi=3.390 \AA$ at $25 \mathrm{meV} / \mathrm{atom}$, which corresponds to the equilibrium spacing and binding energy a graphene bilayer. This has to be compared to the binding energy of $22 \mathrm{meV} / \mathrm{atom}$ at a bilayer separation of $3.32 \AA$ A computed by Gould and co-workers [56] with DFT in the local-density approximation with dispersion corrections. Using a similar calculation, but with an infinite number of layers, we obtain for the equilibrium spacing a value of $d_{\mathrm{C}}=2079^{-1 / 6} \pi \xi$. With the values for $\xi$ and $\varepsilon$ of Che and co-workers [55], the interlayer distance and binding energy of graphite reported by Gould and co-workers are reasonably recovered (3.346 $\AA$ versus $3.34 \AA$ and $57 \mathrm{meV} /$ atom versus $48 \mathrm{meV} / \mathrm{atom}$, respectively). The difference between $d_{\mathrm{C}}^{\min }$ (for the bilayer) and $d_{\mathrm{C}}$ (for infinite graphite) is only of $1.3 \%$, therefore, the spacing of the uppermost layers of a half-space of graphite is expected to be close to the value in bulk graphite.

The summation of Eq. (15) over a semi-infinite number of layers leads to the noncovalent interaction energy between one carbon atom and a half-space of graphitic layers distant by $d_{\mathrm{C}}$,

$$
E_{\mathrm{LJ}, i}^{(\infty)}=\frac{\pi \sigma \varepsilon}{2}\left[\frac{\xi^{12}}{5 d_{\mathrm{C}}^{10}} \zeta\left(10, \frac{d_{i}}{d_{\mathrm{C}}}\right)-\frac{\xi^{6}}{d_{\mathrm{C}}^{4}} \zeta\left(4, \frac{d_{i}}{d_{\mathrm{C}}}\right)\right]
$$

thereby recovering again the results first obtained by Steele [45]. This expression represents an external potential on the only graphitic layer that is explicitly present in the implicit model.

\section{E. Screening of dispersion forces on metal substrates}

Dispersion forces on a metal substrate are screened at long distance owing to electron delocalization [57]. Such nonadditive effects can be empirically accounted for by introducing an additional Thomas-Fermi (TF) screening factor into the Grimme D2 model in the case of the interaction with the metal part of the substrate [58-60]:

$$
\begin{gathered}
E_{\mathrm{disp}}^{\text {(D2,scr. })}=\sum_{i} \sum_{j \neq i} \frac{-s_{6} f_{\mathrm{scr}}\left(d_{i}\right)}{1+\exp \left[-20\left(\frac{r_{i j}}{R_{r}^{i j}}-1\right)\right]} \frac{C_{6}^{i j}}{r_{i j}^{6}}, \\
f_{\mathrm{scr}}\left(d_{i}\right)=\exp \left[-2\left(d_{i} / r_{\mathrm{TF}}\right)\right],
\end{gathered}
$$

where $r_{\mathrm{TF}}$ is the screening length. In the previous equation, the layered structure of the substrate was explicitly taken into account through the additional and translationally invariant dependence on the distance $d_{i}$ between atom $i$ and the layer to which $j$ belongs. Approximating the substrate by a series of equidistant uniform atomic monolayers with density $\sigma^{\prime}$ allows summing over entire layers as before, and the result reads as

$$
\widetilde{E}_{\text {disp }}^{(\text {scr })}=-\frac{s_{6} \sigma^{\prime} C_{6} \pi}{2 d_{L}^{4}} \sum_{i \in \text { ads }} e^{\frac{-2 d_{i}}{r_{\mathrm{TF}}}} L\left(e^{\frac{-2 d_{L}}{r_{\mathrm{TF}}}}, 4, \frac{d_{i}}{d_{L}}\right)
$$

where $L(z, s, a)$ denotes the Lerch zeta function

$$
L(z, s, a)=\sum_{k=0}^{\infty} \frac{z^{k}}{(k+a)^{s}} .
$$

The case without screening of dispersion is recovered for $r_{\mathrm{TF}} \rightarrow \infty$, whereas taking $r_{\mathrm{TF}}=0$ corresponds to removing the dispersion correction entirely. As was the case before, the two uppermost layers must be dealt with separately owing to distances shorter than the cutoff, and Eq. (11) with the additional screening factor has to be used instead. In this expression, the $C_{6}$ and $R_{r}$ parameters must be chosen appropriately for the pair of subsystems in consideration, which can be adsorbate-graphene, adsorbate-metal substrate, or graphene-metal substrate. In addition, $\sigma$ needs to be replaced be $\sigma^{\prime}$ for the second layer. In practice, the Lerch zeta function was evaluated with the lerch function of the POLPAK library [61].

As a side note, we emphasize that Eq. (19) applies to the dispersion interactions exerted by the semi-infinite metallic substrate on both the metal adsorbate and the epitaxial graphene layer (even in absence of any adsorbate).

\section{F. Computational details}

The different dispersion models presented in the previous section have been compared against each other for various adsorbates on several substrates. All simulations employed periodic boundary conditions along the two lateral dimensions. In the static limit, Pt nanoparticles containing between one and several thousand atoms were locally optimized starting from high-symmetry morphologies such as multilayer icosahedra or truncated octahedra (Wulff shapes). For both types, the nanoparticles were initially deposited in epitaxy on the graphite substrate, exposing their (111) facets to the honeycomb lattice. From those structural optimizations, adsorption energies and geometry relaxations could be quantified.

In addition to these static investigations, molecular dynamics simulations at finite temperature were conducted to assess the thermal stability and dynamical properties of adsorbates on their carbonated substrates. Here, we used a combination of methods to simulate the real-time dynamics, essentially molecular dynamics (MD) at finite temperature imposed by coupling a Nosé-Hoover thermostat to the substrate. In order not to alter the time-dependent properties, the thermostatting procedure was not applied to the subsystem of interest (adsorbate or upper layer in the case of epitaxial graphene), but to the remaining substrate only.

MD trajectories for 64-atom NPs on substrates kept at a fixed temperature were initiated from locally relaxed structures occupying a two-layer configuration similar to experimentally observed nanoparticles formed in situ on epitaxial graphene on (111) metal surfaces $[4,7,62,63]$. Various observables were calculated in order to probe the intrinsic mobility of the nanoparticles, their possible diffusion on the substrate as well as their spontaneous rearrangement toward lowerenergy structures. Vibrational spectra were also evaluated from the Fourier transform of the velocity time autocorrelation function. Unless otherwise mentioned, all molecular dynamics trajectories employed a time step of $1 \mathrm{fs}$ and were carried out 
over $5.5 \mathrm{~ns}$, the first $500 \mathrm{ps}$ being discarded from the averages for equilibration purposes.

For the static computations of the smaller Pt adsorbates on graphite, the periodic boundaries contained $15 \times 15$ graphene unit cells, unless otherwise mentioned, and for the larger $\mathrm{Pt}$ adsorbates (from 923 atoms on) $30 \times 30$ unit cells. In the case of the MD simulations of $\mathrm{Pt}_{64}$ clusters on (possibly defective) graphite, a simulation box of $10 \times 10$ graphene unit cells was chosen. It was made sure that these simulation boxes are large enough that the adsorbates do not interact with their periodic images, i.e., the cell width is larger than the diameter of the adsorbate plus the cutoff of the covalent interatomic potential. Regarding epitaxial graphene on $\operatorname{Pt}(111)$, the size of the simulation cell is imposed by the periodicity of the moiré and varies as a function of the in-plane angle between the graphene and the Pt lattice, as discussed in Sec. IV. At large in-plane angles, larger sizes were simulated in order to circumvent the problem of small primitive cells. All calculations using explicit dispersion models such as Grimme D2 and D3 employed three graphene slabs in the case of graphite (ABA fashion), and three metallic slabs in the case of epitaxial graphene on $\mathrm{Pt}(111)$ (ABC fashion) independently of the dispersion model. Rigid boundary conditions were applied to the bottom layer in the MD simulations and local optimizations. In the implicit description of graphite, the rigid boundaries were released and only the uppermost graphene layer was included as it contributes to possible covalent interactions.

In order to determine the ability of the present dispersion models to describe the interaction of such particles on pristine graphite, we define the adsorption energy $E_{\text {ads }}$ of a general adsorbate or nanoparticle (np) on its substrate (sub) through $E_{\text {ads }}=E_{\text {total }}-E_{\text {sub }}-E_{\text {np }}$, where $E_{\text {total }}$ is the energy of the entire system after relaxation, $E_{\text {sub }}$ and $E_{\mathrm{np}}$ the potential energies of the relaxed substrate and nanoparticle, respectively.

\section{PLATINUM NANOPARTICLES ON PURE CARBONATED SUBSTRATES}

The methodology of the previous section was applied and tested for platinum adsorbates on different extended substrates. In this section, we discuss the most straightforward case of purely graphitic supports containing one (graphene) or an infinite number (graphite) of monolayers.

\section{A. Comparison of dispersion models: The adatom case}

The performance of the different interaction models introduced above was first assessed on static properties involving the local relaxation of adsorbates on the substrates. In the simplest case of single $\mathrm{Pt}$ adatoms adsorbed on graphene and graphite, various interaction sites are illustrated in Fig. 2. The alpha and beta sites are found on top of a carbon atom and differ from each other only once multiple graphene layers are taken into account. Such differences are only relevant in the explicit models, however, they were found to be negligibly small and the two sites will be considered as equivalent here. The bridge and hollow positions lie above the midpoint of a $\mathrm{C}-\mathrm{C}$ bond and the center of a six-membered carbon ring, respectively.

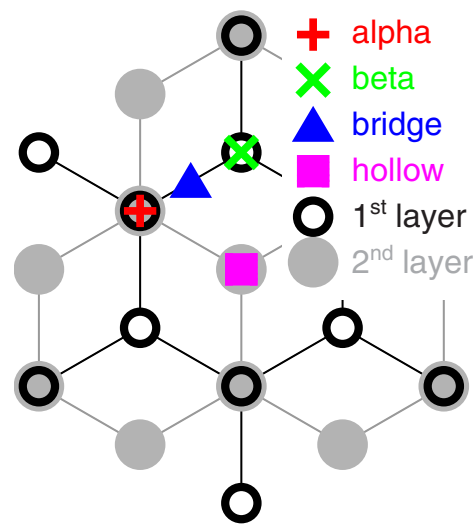

FIG. 2. (Color online) High-symmetry adsorption sites of adatoms on graphite: The atoms of the uppermost graphitic layer are represented as black rings, the atoms of the layer below as gray circles. In the implicit models, the alpha and beta sites are equivalent.

Energy profiles were calculated for Pt adatoms approaching perfectly flat graphene and graphite surfaces containing laterally $10 \times 10$ graphene unit cells vertically at these various sites. Here, the substrate atoms where kept fixed to their optimized positions without adatom. It should be noted that the distance of the adatom to the graphitic layer differs from the smallest Pt-C separation in the case of the bridge and hollow sites.

The variations of the energy profiles with atom-substrate distance are represented in Fig. 3 for the three relevant adsorption sites and for the graphene and graphite substrates, with a reference energy assigned at infinite separation.

For both substrates, correcting for dispersion forces further binds the adatom by $0.5-1 \mathrm{eV}$ depending on the model, and introduces a shallow long-distance van der Waals minimum close to the cutoff of the covalent part of the potential, which is at $2.8 \AA$. This minimum should be considered as a physisorbed state.

Comparing the curves obtained with the Grimme D2 model and the implicit dispersion model, the implicit model appears to work particularly well in this simple test case where the assumption of an uncorrugated substrate surface is strictly satisfied. The minor discrepancies between the Grimme D2 and D3 models can be explained based on the varying coordination of the adatom as it approaches the substrate: adsorption on an alpha/beta site produces a singly coordinated Pt atom, whereas the adatom is twofold and sixfold coordinated at the bridge and hollow sites, respectively. Within the D3 model, higher coordinations tend to decrease the $C_{6}$ coefficients accordingly, which explains the slight attenuation of the van der Waals energy at short distances for hollow sites with respect to the D2 model. Those observations are valid for both substrates, and unsurprisingly the profiles obtained for graphene and graphite look very similar. Further inspection indicates that, as they should, the chemisorbed minima are slightly more bound in graphite by 86,107 , and $130 \mathrm{meV}$ for the alpha, bridge, and hollow sites, respectively, as the result of additional dispersion forces. Those values for the implicit model are consistent with those obtained with the Grimme D2 model (namely, 66, 88, and $111 \mathrm{meV}$ ), the $20-\mathrm{meV}$ difference arising from the neglect of van der Waals forces beyond the simulation supercell in the Grimme approach. 


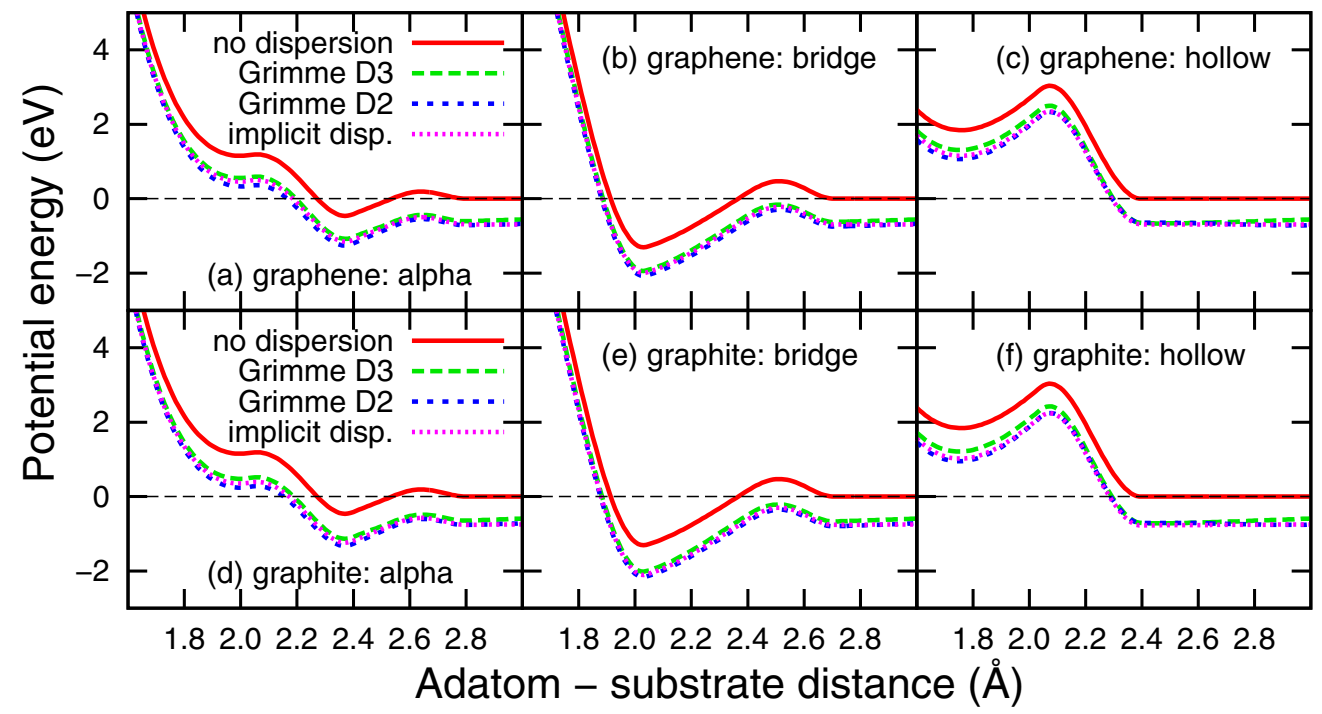

FIG. 3. (Color online) Energy profiles of a Pt adatom approaching flat graphitic surfaces along various sites, as obtained from the bond-order potential corrected with different dispersion models. Upper panels (a)-(c): single-layer graphene; lower panels (d)-(f): semi-infinite graphite.

The main conclusion of Fig. 3 is the very satisfactory performance of the implicit dispersion model to describe the global long-range London forces for Pt atoms on both graphene and semi-infinite graphite, as well as the clear presence of chimisorbed and physisorbed minima separated by well-defined barriers.

\section{B. Pt adatoms on defective graphite}

Defects on surfaces can have a significant influence on the structural and dynamical properties of adsorbates and can be tailored to trap them and even promote self-assembly [64]. The role of defects on the adsorption of a $\mathrm{Pt}$ adatom on a graphitic surface has been investigated with the present explicit (Grimme D2 and D3) and implicit dispersion correction models, as well as without any such correction. Two defects were considered here, namely, a single vacancy and a topological Stone-Wales (5775) defect. For both defects, the graphitic surfaces were locally reoptimized, and the adsorption of a Pt adatom above the defective substrate was monitored as a function of its lateral position, the distance of the adatom perpendicular to the surface being optimized in the process (but at fixed substrate geometry). For the static computations of $\mathrm{Pt}$ adatoms on defective graphite, a simulation box containing $5 \times 5$ graphene unit cells was employed.

The resulting energy maps obtained with the explicit D2 and the implicit dispersion models are shown in Fig. 4 for both defects. In the case of the vacancy, a strong minimum is found away from the uppermost graphitic layer, originating from the longer Pt-C bond compared to C-C bonds. For the Stone-Wales defect, four equivalent energy minima denoted A in Fig. 4(c) are found at a bridge position of the seven-membered rings connecting to hexagons, and four other equivalent minima denoted $\mathrm{B}$ can be defined at the alternative bridges connecting to pentagons.

To better evaluate the relative energies of the various adsorption sites, full relaxations of the substrate and adatom were carried out with the D2, D3, and implicit dispersion corrections. The results of these optimizations for the adsorption energy and lowest $\mathrm{Pt}-\mathrm{C}$ distance are summarized in Table I.

Regarding the Stone-Wales defect, site B turns out to be the most stable for all dispersion correction models. As was the case for adsorption on perfect substrates, additional dispersion in graphite relative to graphene further strengthens the interaction energies in presence of defects, by about $0.1 \mathrm{eV}$ according to the implicit dispersion model. Based on those results, defects of both types at the uppermost graphite layer are expected to bind Pt adsorbates more strongly than pristine graphite.
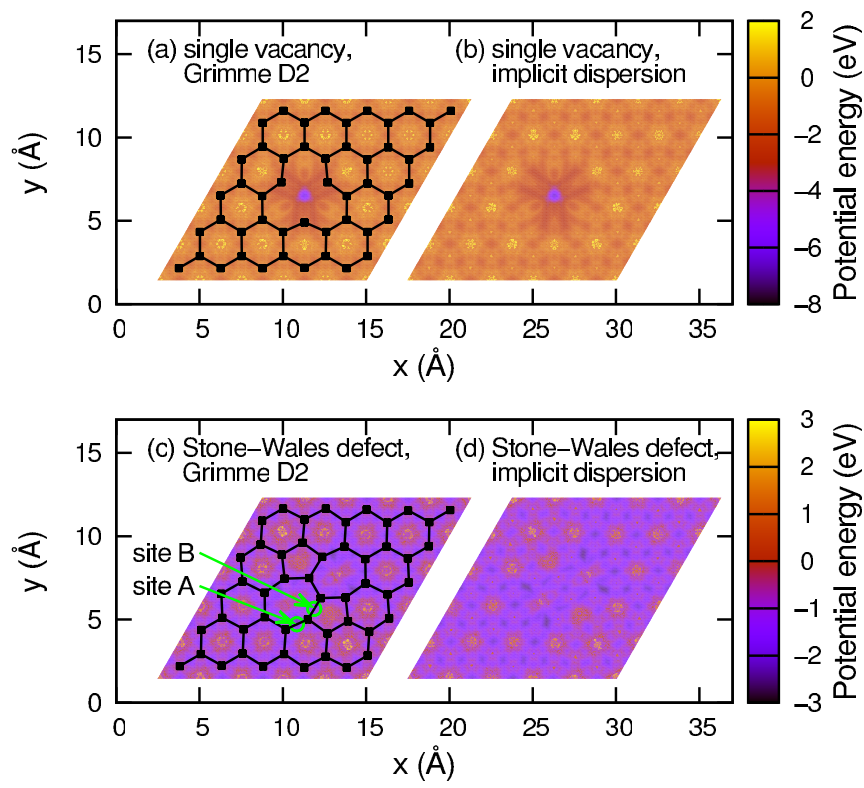

FIG. 4. (Color online) Potential energy of a Pt adatom on a graphite surface with a single vacancy (upper panel) or a 5775 Stone-Wales defect (lower panel) as obtained with the explicit D2 and implicit dispersion models. The defective carbon lattice is explicitly shown for the D2 model. For the Stones-Wales defect, two adsorption sites $\mathrm{A}$ and $\mathrm{B}$ are highlighted as defined in the text. 
TABLE I. Adsorption of a $\mathrm{Pt}$ adatom on defective graphite: adsorption energy $E_{\mathrm{ads}}$ and closest distance $d_{\mathrm{C}, \mathrm{Pt}}$ between the Pt atom and $\mathrm{a}$ atom. In the case of the Stones-Wales defect, adsorption sites $\mathrm{A}$ and $\mathrm{B}$ are indicated in Fig. 4(c).

\begin{tabular}{cccc}
\hline \hline & $\begin{array}{c}\text { Single } \\
\text { vacancy }\end{array}$ & $\begin{array}{c}\text { Stone-Wales } \\
\text { site A }\end{array}$ & $\begin{array}{c}\text { Stone-Wales } \\
\text { site B }\end{array}$ \\
\hline No dispersion & & & \\
$E_{\text {ads }}(\mathrm{eV})$ & -6.46 & -2.61 & -2.63 \\
$d_{\mathrm{C}, \mathrm{Pt}}(\AA)$ & 1.97 & 2.04 & 2.05 \\
Grimme D2 & & & \\
$E_{\text {ads }}(\mathrm{eV})$ & -7.10 & -2.92 & -3.06 \\
$d_{\mathrm{C}, \mathrm{Pt}}(\AA)$ & 1.98 & 2.05 & 2.06 \\
Grimme D3 & & & -2.91 \\
$E_{\text {ads }}(\mathrm{eV})$ & -7.18 & -2.76 & 2.05 \\
$d_{\mathrm{C}, \mathrm{Pt}}(\AA)$ & 1.97 & 2.05 & -3.38 \\
Implicit model & & & 2.05 \\
$E_{\text {ads }}(\mathrm{eV})$ & -7.44 & -3.33 & \\
$d_{\mathrm{C}, \mathrm{Pt}}(\AA)$ & 1.97 & 2.04 & \\
\hline \hline
\end{tabular}

\section{Interaction of larger adsorbates}

Metal nanoparticles can be prepared in the gas phase before their subsequent transfer on substrates [65-68]. Such particles can be quite large, and sustain a much more significant binding to the substrate than single adatoms. Regular icosahedral and truncated octahedral (Wulff) nanoparticles display (111) facets that make them highly stable [69] and compatible with epitaxial deposition on ordered graphite substrates, although such a deposition involves some notable strain owing to the different lattice constants in platinum (bond distance of $2.8 \AA$ ) and in carbon $(2.5 \AA)$. Figure 5 shows the adsorption energy of such nanoparticles deposited on graphene and graphite and locally optimized from an initial configuration with the metal atoms lying on top of the most stable bridge sites, using different dispersion models. Adsorbates containing between 13 and 3871 atoms, or between 1 and 11 icosahedral shells and as many as 6 truncated octahedral shells (2406 atoms) were considered here. In the original bond-order potential without any dispersion correction, only the single adatom and the 13-atom icosahedron appear stable. The chemical interaction with the substrate distorts $\mathrm{Pt}_{13}$ significantly, the mutual distances between the three atoms in contact with the uppermost graphitic layer being increased from $2.7 \AA$ for the free $\mathrm{Pt}_{13}$ to $3.3 \AA$ upon deposition. Similar deformations are found if deposition occurs on alpha/beta sites, however, no major distortion occurs at the hollow site. Those conclusions are essentially unchanged once dispersion corrections are accounted for. For this small 13-atom cluster, the rather large distortion leads to a relatively small adsorption energy when compared to the larger nanoparticles because most of the interaction energy is consumed for deformation (about $42 \%$ for the Grimme D2 and $55 \%$ for the implicit model as compared to an average of $13 \%$ and $14 \%$ for larger icosahedra). The closest distance between the adsorbate and the substrate is in the 2.0-2.1 $\AA$ range in the case of the adatom and $\mathrm{Pt}_{13}$. This distance increases up to slightly below the cutoff of the Pt-C Brenner potential for the larger adsorbates, as a response to the repulsive part of the covalent potential close to the cutoff.

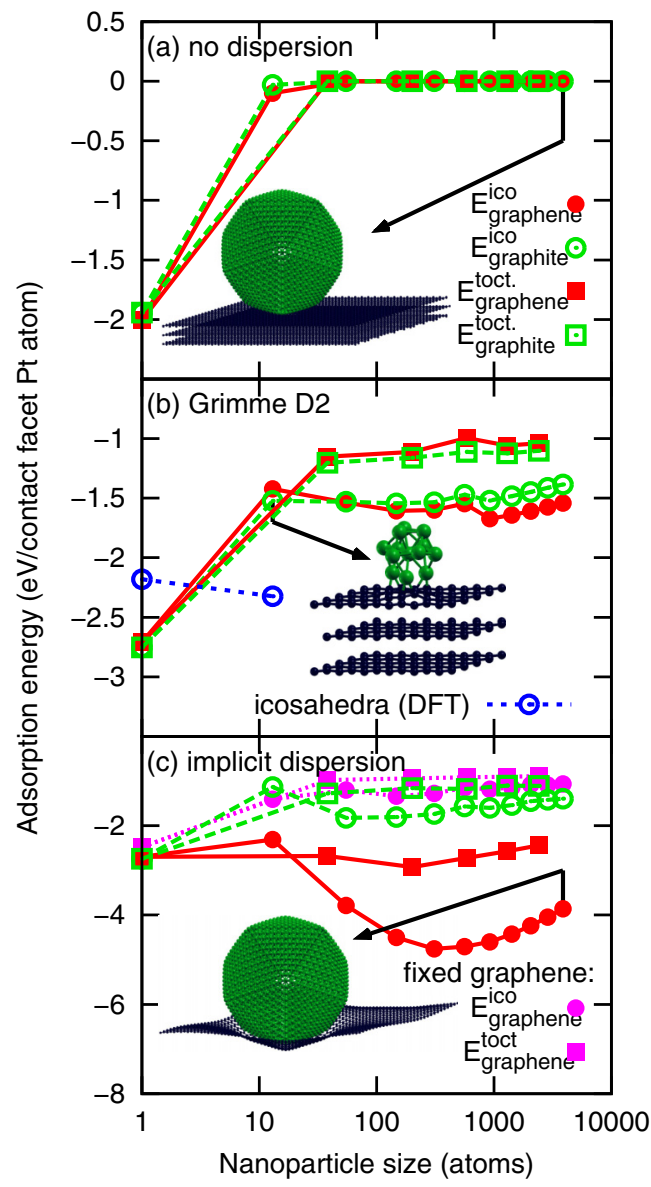

FIG. 5. (Color online) Adsorption energies of Pt nanoparticles on graphene and graphite, computed for different treatments of London dispersion forces, and normalized by the number of metal atoms in contact with the substrate. (a) No dispersion correction; (b) explicit correction of the Grimme D2 type; (c) implicit dispersion model. The adsorption energies, as defined in the text, for graphene and graphite are denoted by full and empty symbols, respectively. Icosahedral (ico) and truncated octahedral (toct) adsorbates are indicated by circles and squares, respectively. DFT data from Ref. [43] have been superimposed in panel (b).

Epitaxial alignment on the bridge sites is essentially lost for the larger clusters, with a slight preference for the hollow site in the case of the 55-atom icosahedron. As the nanoparticles become larger, the mismatch in the lattice constants between $\mathrm{Pt}(111)$ and graphite (0001) results in some excessive strain that the nanoparticles cannot accommodate. For these larger clusters, the adsorption energy is roughly proportional to the number of atoms at the facet in contact with the substrate. The slight weakening in adsorption seen in Figs. 5(b) and 5(c) is due to the decreasing fraction of less coordinated atoms at the edge of the facet in contact. We attribute the weaker interaction per interface atom in truncated icosahedra to their higher area exposed in contact with the substrate. If the implicit dispersion model uses the average position $\bar{z}$ from the carbon atoms in the simulation cell, the planar approximation may break down for large adsorbates due to excessively large deformations and the tendency to minimize the global interaction. This in turns introduces some dependence of the results on the 
size of the simulation supercell. For this reason, a large box of $30 \times 30$ graphene unit cells has been used for the implicit dispersion model. This issue, which is illustrated in the inset of Fig. 5(c), can be tackled by fixing vertically the graphene layer during the minimizations. Repeating the minimizations with the implicit model under this constraint leads to better behaved adsorption energies also shown in Fig. 5(c), however, the need for this trick clearly indicates that the implicit models should be reworked for large nanoparticles on graphene, despite their correct behavior for graphite owing to the interlayer repulsion. One straightforward correction specific to graphene could be to have the implicit model only act beyond the simulation box (i.e., to account for the nonprimitive cells only), and keep an explicit description within the primitive cell, which incidentally would not cause a large computational overhead since the relevant distances are needed for the covalent/metallic contributions anyway.

For graphite, good agreement between Figs. 5(b) and 5(c) is reached independently of adsorbate size, indicating that the approximations underlying the implicit model are well fulfilled. In particular, the substrate corrugation due to the interaction with the deposited nanoparticle remains moderate enough for the assumption of a planar upper layer to remain valid. For the adsorption on graphene, the agreement between the implicit and the Grimme D2 dispersion model is not as good due to high corrugation. As will be seen in the following from our dynamical simulations, significant structural rearrangements take place already in small adsorbates, and it is rather unclear as to what the optimal shape of the nanoparticles should be in such large systems deposited on nonrigid substrates.

\section{Comparison with DFT calculations}

The predictions of the present semiempirical models can be compared against available electronic-structure reference data obtained at the level of density-functional theory calculations. Ramos-Sanchez and Balbuena [43] investigated the interaction of a Pt adatom and small Pt adsorbates deposited on various sites of graphite by means of dispersion-corrected DFT (RPBE-D2). The adsorption energy for the adatom found by these authors amounts to $-2.18 \mathrm{eV}$ at the bridge site with a shortest Pt-C bond of $2.03 \AA$, both quantities being in satisfactory agreement with the present calculations where values of $-2.74 \mathrm{eV}$ (implicit model) and $-2.75 \mathrm{eV}$ (Grimme D2) are, respectively, obtained at a common closest Pt-C distance of $2.06 \AA$.

In the case of graphene, several other DFT data without dispersion corrections have been reported for the adatom, but the values are significantly spread out. The adsorption energies for a Pt adatom at the most stable bridge position thus range from -0.37 [70] to -1.5 [71], -1.57 [72], -2.03 [73], -2.17 [74], and $-3.2 \mathrm{eV}$ [75], with equilibrium distances that always exceed the values obtained with dispersion corrections, although the variations among the different authors are much smaller $(2.148,2.101,2.10,2.12,2.08$, and $2.15 \AA$, respectively).

Results for the icosahedral $\mathrm{Pt}_{13}$ adsorbate are much more scarce, however, Ramos-Sanchez and Balbuena [43] reported an adsorption energy of $-6.97 \mathrm{eV}$ at the bridge site, which is noticeably larger than the values obtained here of
$-4.27 \mathrm{eV}$ and $-3.40 \mathrm{eV}$ in the explicit and implicit dispersion models. Using a finite cluster model of the graphene substrate, Okamoto [76] computed an adsorption energy of -1.08 and $-2.08 \mathrm{eV}$ at two other adsorption sites, but with the same orientation of $\mathrm{Pt}_{13}$ on a graphene sheet. Additional densityfunctional results are available in the case of $\mathrm{Pt}$ adatoms on defective graphene [72,77]. Our values for the adsorption of a Pt adatom on a vacancy in graphite reported in Table I are comparable to the adsorption energy of a $\mathrm{Pt}$ atom on a vacancy in graphene of $-7.45 \mathrm{eV}$ at a Pt-C distance of $1.94 \AA$ calculated at the DFT level without dispersion correction by Fampiou and co-workers [72]. These authors further reported an adsorption energy of $-6.68 \mathrm{eV}$ obtained with the Albe potential at $1.98 \AA$ Pt-C separation, which is in good agreement with our own values for graphite. Repeating the calculations for defective graphene yields an adsorption energy of $-7.37 \mathrm{eV}$ at $1.98 \AA$ separation. The difference with the aforementioned semiempirical energy of Fampiou et al. thus measures the additional dispersion interaction beyond the simulation cell.

Other adsorption energies for larger Pt adsorbates containing up to 27 atoms have been reported by Qi et al. from DFT calculations without dispersion corrections [77]. Unfortunately, comparison with those results is not straightforward because the structures considered by those authors differ significantly from ours.

\section{E. Dynamics of adsorbates on graphite}

One essential advantage of analytical potentials over methods based on an explicit description of electronic structure is their ability to address physical properties on much stronger statistical grounds. Here, we discuss the thermal stability of two-layered $\mathrm{Pt}_{64}$ nanoparticles on graphitic substrates, using molecular dynamics trajectories spanning the nanosecond regime. Two indices were defined to monitor the dynamical evolution of the adsorbates as a function of increasing temperature, based on the root-mean-square bond length fluctuation of selected atomic pairs. The intrinsic rigidity within the particles can be measured by

$$
\delta_{\text {intra }}=\frac{1}{N(N-1)} \sum_{i \in \text { ads }} \sum_{\substack{j \in \text { ads } \\ j \neq i}} \frac{\sqrt{\left\langle r_{i j}^{2}\right\rangle-\left\langle r_{i j}\right\rangle^{2}}}{\left\langle r_{i j}\right\rangle},
$$

where $N$ is the number of atoms in the adsorbate and $\langle\ldots\rangle$ a time average. Similarly, the global mobility of the adsorbate relative to its substrate (subst) can be evaluated by the index

$$
\delta_{\text {inter }}=\frac{1}{N \times M_{1}} \sum_{i \in \text { ads }} \sum_{\substack{\text { st subst } \\ 1^{\text {stlayer }}}} \frac{\sqrt{\left\langle r_{i j}^{2}\right\rangle-\left\langle r_{i j}\right\rangle^{2}}}{\left\langle r_{i j}\right\rangle},
$$

$M_{1}$ denoting the number of atoms in the upper layer of the substrate.

At low temperature, where both the substrate and adsorbate vibrate around their equilibrium positions, the interatomic distances only exhibit some minor fluctuations and the two indices keep low values, typically below a few percent. Conversely, an index exceeding approximately 10\%-15\% indicates some qualitative motion of the atoms within the 


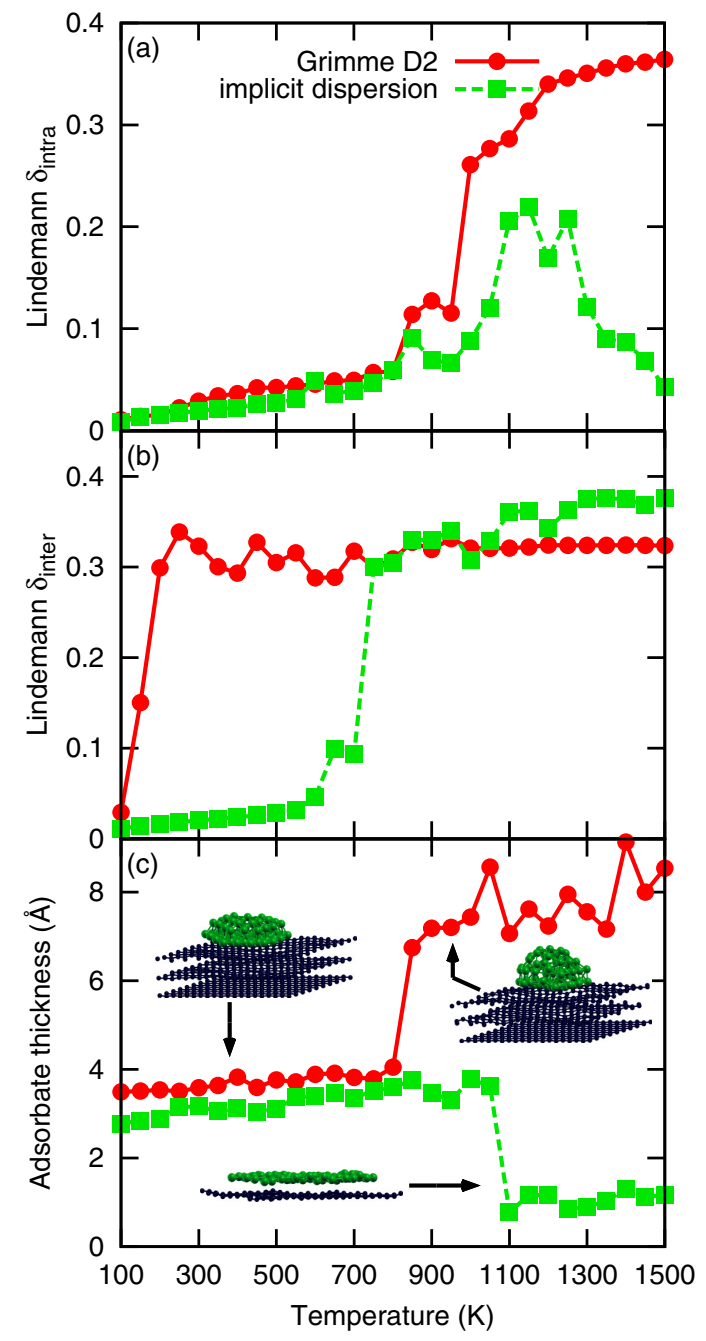

FIG. 6. (Color online) Temperature variations of the root-meansquare bond length fluctuation indices measuring the internal rigidity of the $\mathrm{Pt}_{64}$ adsorbate ( $\delta_{\text {intra }}$ ) and the mobility of the adsorbate relative to the graphite substrate $\left(\delta_{\text {inter }}\right)$, and average adsorbate thickness $\sigma$ as obtained from the explicit (Grimme D2) and implicit dispersion models.

adsorbate (fluxional or liquid state detected on $\delta_{\text {intra }}$ ) or relative to the substrate (global diffusion detected on $\delta_{\text {inter }}$ ).

Figures 6(a) and 6(b) show the variations of these order parameters with increasing temperature for the example of the $\mathrm{Pt}_{64}$ adsorbate assumed to initially occupy a bilayer shape in near epitaxy with the graphite substrate at low temperature. The simulations were performed using the explicit (D2) and implicit models of dispersion corrections. Simulations neglecting dispersion corrections altogether were also performed, but produced unphysical results with the spontaneous desorption of the adsorbate already below room temperature. This behavior was actually anticipated as it is consistent with the vanishing adsorption energy of clusters larger than 13 atoms unless dispersion forces are accounted for [see Fig. 5(a)]. For both dispersive correction models, the adsorbed nanoparticles undergo increasingly large vibrations as temperature is increased, and a progressive but clearly visible transition to a more disordered state with $\delta_{\text {intra }}>0.15$ near $1000-1200 \mathrm{~K}$. However, the other index $\delta_{\text {inter }}$ reveals that the dynamics of the adsorbate on the substrate depends very sensitively on the model. In the explicit D2 description, the nanoparticle moves relative to the substrate already above $200 \mathrm{~K}$, whereas $750 \mathrm{~K}$ are necessary for such a motion to take place in the implicit model. These qualitative differences are quite significant, and reveal some contrasted energetic behaviors between the two approaches. We have examined the structural evolution of the adsorbate by calculating its geometrical extension (or thickness) $\sigma$ perpendicular to the substrate. Starting from a bilayer particle with $\sigma \approx 2.7-4.1 \AA$, the variations of $\langle\sigma\rangle(T)$ with temperature are shown in Fig. 6(c).

The different thermodynamical behaviors can now be related to different variations in the shape of the adsorbate, which becomes more spherical in the explicit D2 description $(\langle\sigma\rangle \sim 8 \AA)$, but conversely much flatter $(\langle\sigma\rangle \sim 1 \AA)$ if the dispersion correction is coarse grained in the implicit model. The structural transitions here simply originate from the lower energy of those structures in their respective models, and the different temperatures of their onsets found in the variations of $\langle\sigma\rangle(T)$ roughly match the regions where $\delta_{\text {intra }}$ increases in Fig. 6(a), albeit more smoothly due to the more regular one-layer structure of the adsorbate. Local minimization of the high-temperature structures confirms that the ellipsoidal isomer is much more stable in the explicit D2 model, by $5.6 \mathrm{eV}$, the flat isomer conversely lying $14.5 \mathrm{eV}$ below the initial structure in the implicit dispersion model. These results motivate more systematic work on global optimization, which would shed more light onto the wetting behavior of the different dispersion models but lie beyond the present effort.

Although the present trajectories were carried out of equilibrium from a metastable state, they were able to locate low-energy minima shedding light onto different binding properties of the present dispersion models. The greater extent of wetting obtained with the implicit model thus reflects a stronger London interaction that is even comparable in magnitude to the metallic contribution holding the adsorbate together. From the purely energetic perspective, the dispersion corrections in the implicit model were indeed expected to be stronger, simply because the explicit approach neglects the contributions from the semi-infinite substrate beyond the atoms from the simulation cell. What Fig. 6(b) further shows is that this stronger pinning of the adsorbate to the substrate also has dynamical consequences, the ability to diffuse being significantly suppressed. That the most stable structures of Pt adsorbates are so significantly affected by the nature and magnitude of dispersion interactions was itself not so obvious a priori, and constitutes one major finding of this work that confirms the importance of those "corrections" on the global potential energy surface.

The imperfect nature of the epitaxial contact between the metal adsorbate and the substrate causes some jiggling motion that was further investigated in details by following not only the center of mass, but also the global orientation of the adsorbate relative to the substrate. More precisely, we evaluated the angle $\theta$ between the substrate and some reference axes of the adsorbate, defined by symmetry in the reduced range $0 \leqslant \theta \leqslant 30^{\circ}$, as a measure of alignment between the two lattices. A value $\theta=0$ corresponds to perfect 


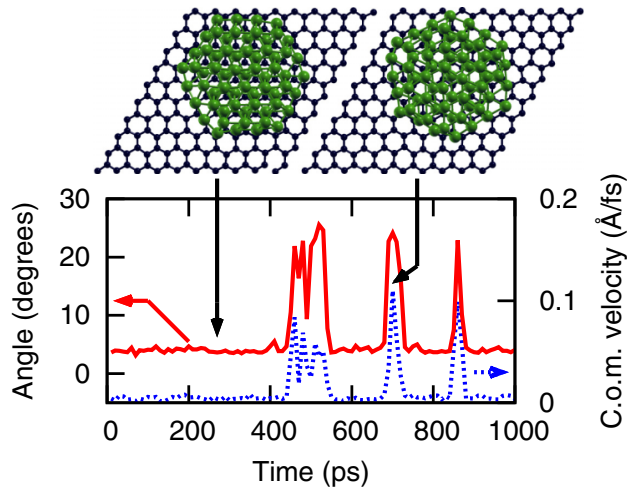

FIG. 7. (Color online) Short-time average angle between the $\mathrm{Pt}_{64}$ adsorbate and substrate lattices, and center-of-mass velocity of the adsorbate, as obtained from a MD trajectory at $900 \mathrm{~K}$ using the implicit model for dispersion corrections. Adsorbates under maximal (left) and minimal (right) alignment are also depicted.

epitaxial alignment, whereas maximal orientational mismatch is attained for $\theta=30^{\circ}$. In the MD simulations, snapshots were taken every picosecond and the time series $\theta(t)$ was analyzed.

At low temperature, the particle is stable on the substrate and vibrates. At sufficiently high temperature, the diffusion motion starts to take place through some occasional jumps and jiggling. Figure 7 illustrates the origin of this jiggling motion on a typical time series $\theta(t)$ obtained for a trajectory at $900 \mathrm{~K}$, together with the corresponding effective velocity of the center of mass of the adsorbate. The simulations were performed here with the implicit dispersion model. At this temperature, most of the time is spent in good alignment between the adsorbate and the substrate, but misalignment occurs on short time scales and in correlation with sudden increases in the velocity modulus. Crossing these energy barriers gives the adsorbate enough velocity to travel rather long distances, the diffusion process showing properties similar to those of a (truncated) Lévy flight [78] with long residences between shorter jumps. Such processes have already been identified for nanoparticle diffusion on surfaces [79-81].

Defects on surfaces can significantly alter the diffusion properties of adsorbates [82]. The influence of the vacancy and Stone-Wales defects in the uppermost graphitic layer on the diffusion propensity of $\mathrm{Pt}_{64}$ adsorbates was also addressed by MD simulations, using the implicit dispersion correction model. The results (not shown) indicate only a modest slowing down in the dynamics that amounts to a few percent reduction in the diffusion constant.

In addition to the long-time diffusion dynamics, the MD trajectories provide a wealth of information about the vibrational dynamics occurring on shorter time scales. The vibrational spectrum was calculated from the Fourier transform of the velocity time autocorrelation function $C_{v v}(t)=\langle\vec{v}(t) \cdot \vec{v}(0)\rangle$ accumulated over windows of 4 ps from 1-ns-long trajectories. While the formula for $C_{v v}(t)$ involves a further average over all atoms, the specific contributions of the adsorbate and substrate can also be evaluated separately.

At low temperature, the system should behave as a collection of harmonic oscillators combining the phonons of the substrate and the normal modes of the adsorbate. The harmonic

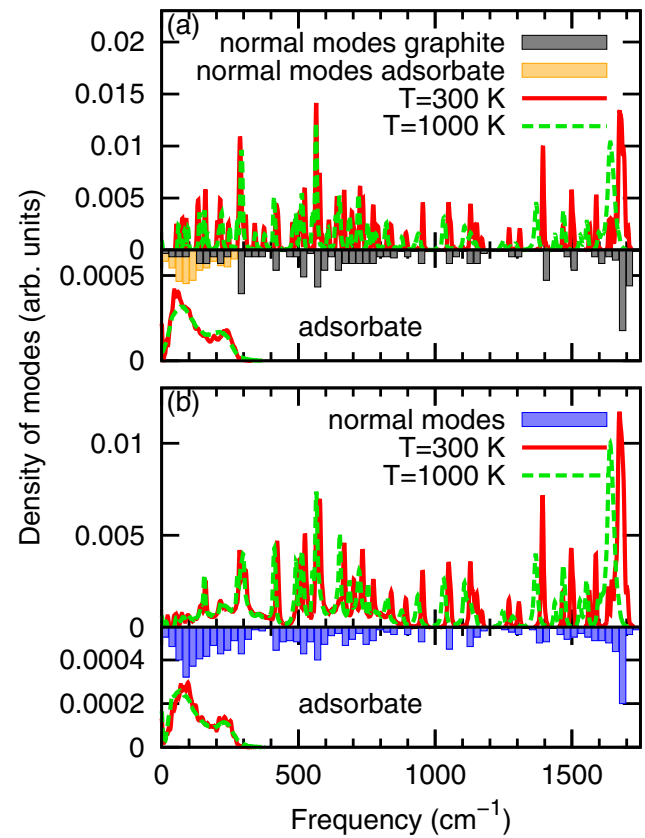

FIG. 8. (Color online) Vibrational spectra for a two-layer $\mathrm{Pt}_{64}$ adsorbate on graphite obtained from the velocity time correlation function at finite temperatures (continuous lines) and in the harmonic limit (histograms pointing downward). (a) Spectra of isolated substrate (upper part) and adsorbate (lower part); (b) global spectra of the entire system (upper part) and of adsorbate alone (lower part) but interacting with the substrate. The calculations were performed with the implicit dispersion correction model.

vibrational frequencies of the global system were calculated from the diagonalization of the mass-weighted Hessian matrix at the locally relaxed position. Here, the second derivatives were obtained by numerical differentiation of the analytical gradient. The harmonic spectrum is expected to resemble the vibrational spectrum determined from the MD simulations only at low temperature, hence the comparison between the two types of calculations provides direct insight into anharmonicities and their manifestations at finite temperature in terms of band shifting and broadening.

The implicit dispersion model was again used for those static and dynamical calculations, the simulations at finite temperature being performed at 300 and 1000 K. Figure 8(a) shows the vibrational spectra of the free graphitic surface and the free two-layered $\mathrm{Pt}_{64}$ at these temperatures as well as the normal mode frequencies of these two systems. All spectra exhibit similar features, with intense lines corresponding to grouped modes at low frequencies and reaching about $1700 \mathrm{~cm}^{-1}$. The far-IR range corresponds to the frequencies of the metal adsorbate, which fall into a rather continuous range below $350 \mathrm{~cm}^{-1}$. For the adsorbate itself, deposition on graphite does not alter the vibrational properties very markedly. In contrast, the graphite substrate undergoes a more significant perturbation due to the adsorbate with clear band broadening and even merging of many small peaks up to $700 \mathrm{~cm}^{-1}$. As expected, the spectral features corresponding to stretching $\mathrm{C}-\mathrm{C}$ modes at higher frequencies are less affected. The perturbation exerted by the adsorbate on the vibrations of the graphitic layers appears more clearly at finite temperature, 


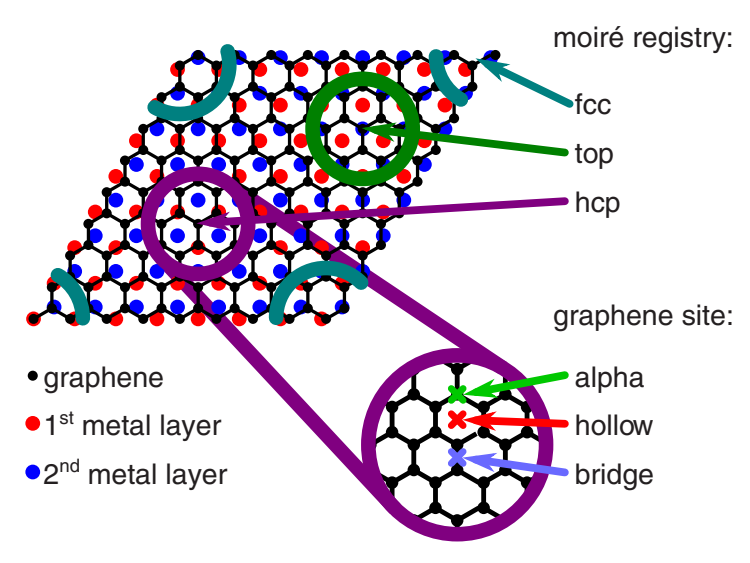

FIG. 9. (Color online) Inventory of moiré sites of the graphene/Pt(111) system with a vanishing in-plane angle ( $\xi$ structure).

and we speculate that the extended range of these perturbations results from hot and combination bands.

Anharmonicities are also evident on the highest frequency band near $1600 \mathrm{~cm}^{-1}$, which displays some slight broadening but more importantly some red-shifting as temperature varies from 0 to $300 \mathrm{~K}$ and finally $1000 \mathrm{~K}$. This peak, which dominates the $\mathrm{G}$ band of graphite, has been further quantified below in the case of epitaxial graphene.

\section{NANOPARTICLES ON GRAPHENE SUPPORTED ON METAL}

The implicit dispersion correction model has been extended to describe epitaxial graphene on $\mathrm{Pt}(111)$, and Pt adsorbates on such substrates. Epitaxial graphene [83] on platinum has been studied by atomic force microscopy [84] and by scanning tunneling microscopy $[85,86]$ possibly coupled to other techniques such as local tunneling barrier height [87] or low-energy electron diffraction [88,89]. Although usually synthesized by chemical vapor deposition, epitaxial graphene on $\mathrm{Pt}(111)$ has also been produced by colliding carbonated molecules such as methane [90] or $\mathrm{C}_{60}$ [91], by subsurface segregation [92], and by liquid phase deposition [93].

Epitaxial graphene on metal forms moiré structures with large periodicities resulting from the lattice mismatch between graphene and the underlying metal $[83,94]$. Such nanomeshes could be used as templates for depositing nanoparticles in a stable fashion $[4,68,95]$.

\section{A. Modeling of supported graphene}

The lattice constant of graphene is about $\frac{1}{8}$ smaller than the equilateral triangular surface lattice of $\operatorname{Pt}(111)$ and therefore a moiré pattern can be produced if the two lattices are superimposed under the commensurate $\frac{9}{8}$ size ratio. In comparison to other transition metals such as nickel, graphene interacts with platinum rather weakly, which further allows additional moiré patterns with different periodicities to be formed by varying the in-plane angle $\Theta$ between the two lattices [83].

Figure 9 depicts the geometry of a typical moiré structure formed by epitaxial graphene and the relevant adsorption sites resulting from these two commensurate lattices in contact with

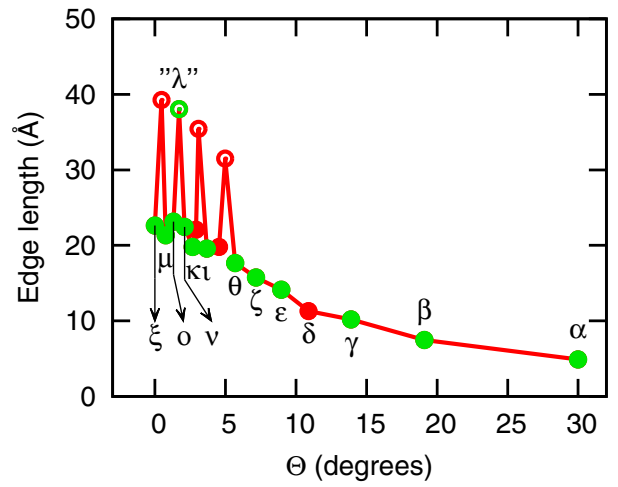

FIG. 10. (Color online) Edge length of moiré structures obtained for graphene on $\mathrm{Pt}(111)$ by varying the in-plane angle between the two lattices obtained by geometric construction (red circles) and observed in experiments (green circles, see also Table II for the assignment of the different structures). Full and empty circles refer to simple commensurate lattices and to commensurate lattices of twice the size of the apparent moiré periodicity, respectively.

each other. In addition to the aforementioned alpha, bridge, and hollow adsorption sites on bare graphene, there are several regions of alignment or misalignment between the two lattices. In the fcc region, the carbon atoms are on top of Pt atoms of either the first or second Pt layer. In the top region, the center of the six-membered carbon rings is on top of the Pt atoms of the uppermost layer. Finally, the hcp region has those rings aligned with the atoms of the second Pt layer.

The nature and properties of moiré structures largely depend on geometrical factors, periodicity being intimately related to commensurability. Several authors have addressed these issues in the specific case of epitaxial graphene [96,97]. A dedicated procedure was implemented here to systematically determine possible moiré structures as a function of the in-plane angle, by looking numerically for close concordances between the two lattices. Again, owing to the symmetry of the problem $\Theta$ was varied between $0^{\circ}$ and $30^{\circ}$ only. In practice, once such a close concordance was detected, the in-plane angle was precisely adjusted and the graphene layer was stretched or squeezed in order to match the $\operatorname{Pt}(111)$ lattice. This way, all possible moiré structures up to a certain predefined size can be listed. The edge length of the supercell obtained by this purely geometrical procedure is illustrated in Fig. 10. The general decrease in the edge length of the moiré supercell with increasing $\Theta$ is equivalent to an increase in periodicity (full circles in Fig. 10), except at low angles where the monotonic relation does not hold and where the periodicity is better represented by an approximate half-value (empty circles in Fig. 10). The minor deviations from monotonic behavior arise from the slight adjustment made to graphene to match the common cell size.

A similar numerical approach for the determination of moirés was followed earlier by Merino and co-workers [91]. The names assigned by these authors to some specific structures are detailed in Table II and are also indicated in Fig. 10. Near the angle of $1.71^{\circ}$ a structure very similar to the $\lambda$ moiré reported by Merino et al. was found but with twice 
TABLE II. Moiré patterns obtained from geometrical considerations, referred to using Wood's notation for the geometry with respect to graphene and ordered with increasing in-plane angle $\Theta$. When applicable, the name assigned to the structure by Merino and co-workers [91] is also reported. References where the corresponding structures have been studied for graphene on $\mathrm{Pt}(111)$ are given in the last column.

\begin{tabular}{lcll}
\hline \hline Moiré in Wood's notation & $\Theta\left(^{\circ}\right)$ & Name [91] & References \\
\hline$(9 \times 9) \mathrm{R} 0.00^{\circ}$ & 0.00 & $\xi$ & {$[85,88,91,93]$} \\
$(\sqrt{244} \times \sqrt{244}) \mathrm{R} 26.33^{\circ}$ & 0.46 & & \\
$(\sqrt{73} \times \sqrt{73}) \mathrm{R} 5.82^{\circ}$ & 0.77 & $\mu$ & {$[84,91]$} \\
$(\sqrt{84} \times \sqrt{84}) \mathrm{R} 10.89^{\circ}$ & 1.32 & $\mathrm{o}$ & {$[91]$} \\
$(\sqrt{229} \times \sqrt{229}) \mathrm{R} 16.63^{\circ}$ & 1.71 & $“ \lambda ”$ & \\
$(\sqrt{79} \times \sqrt{79}) \mathrm{R} 17.00^{\circ}$ & 2.11 & $v$ & {$[91]$} \\
$(\sqrt{63} \times \sqrt{63}) \mathrm{R} 19.11^{\circ}$ & 2.68 & $\kappa$ & {$[84,91]$} \\
$(\sqrt{76} \times \sqrt{76}) \mathrm{R} 23.41^{\circ}$ & 2.92 & & \\
$(\sqrt{199} \times \sqrt{199}) \mathrm{R} 7.05^{\circ}$ & 3.09 & & \\
$(\sqrt{61} \times \sqrt{61}) \mathrm{R} 26.33^{\circ}$ & 3.67 & $\iota$ & {$[84,87,91]$} \\
$(\sqrt{61} \times \sqrt{61}) \mathrm{R} 26.33^{\circ}$ & 4.54 & & \\
$(\sqrt{157} \times \sqrt{157}) \mathrm{R} 3.96^{\circ}$ & 4.99 & & {$[91]$} \\
$(7 \times 7) \mathrm{R} 21.79^{\circ}$ & 5.69 & $\theta$ & {$[91]$} \\
$(\sqrt{39} \times \sqrt{39}) \mathrm{R} 16.10^{\circ}$ & 7.15 & $\zeta$ & {$[84,91]$} \\
$(\sqrt{31} \times \sqrt{31}) \mathrm{R} 8.95^{\circ}$ & 8.95 & $\varepsilon$ & {$[88,91]$} \\
$(\sqrt{21} \times \sqrt{21}) \mathrm{R} 10.89^{\circ}$ & 10.89 & $\delta$ & {$[85,88,91,98]$} \\
$(4 \times 4) \mathrm{R} 0.00^{\circ}$ & 13.90 & $\gamma$ & $\beta$ \\
$(3 \times 3) \mathrm{R} 0.00^{\circ}$ & 19.11 & $\beta$ & $\alpha$ \\
$(2 \times 2) \mathrm{R} 0.00^{\circ}$ & 30.00 & $\alpha$ & \\
\hline \hline
\end{tabular}

the size of the super cell. This structure is denoted as " $\lambda$ " in Table II.

Using the present potentials, the moire structures were locally optimized in order to determine the magnitude of corrugation $\Delta z$ of the graphene layer and its separation with the metal. The adsorption energies averaged over all moiré structures and obtained with the Grimme D2 model (334 meV/carbon atom) or the implicit dispersion model (355 meV/carbon atom) are very similar to each other but significantly overestimate the recent predictions of $43 \mathrm{meV} / \mathrm{carbon}$ atom by Vanin and co-workers [99] based on vdW-DFT calculations, which satisfactorily agree with earlier computations by Khomyakov and co-workers ( $38 \mathrm{meV} / \mathrm{carbon}$ atom for the $\alpha$ moiré [98]) and by Gao et al. (38.6 and $39.8 \mathrm{meV} /$ carbon atom for the $\xi$ and $\alpha$ moirés, respectively [88]).

The overestimation with the present models is due to the absence of screening in both explicit and implicit dispersion models, which is important owing to the delocalized nature of the electron cloud in the bulk metal. One empirical way of accounting for these screening effects consists of considering the contributions to the London interactions of the uppermost layer only $[16,100]$. Repeating the calculations under such conditions leads to a significantly reduced adsorption energy of $273 \mathrm{meV} /$ carbon atom, still off by a factor 6 with respect to the reference data of Vanin et al. [99], the uppermost metal layer alone contributing to about $77 \%$ of the total dispersion energy.

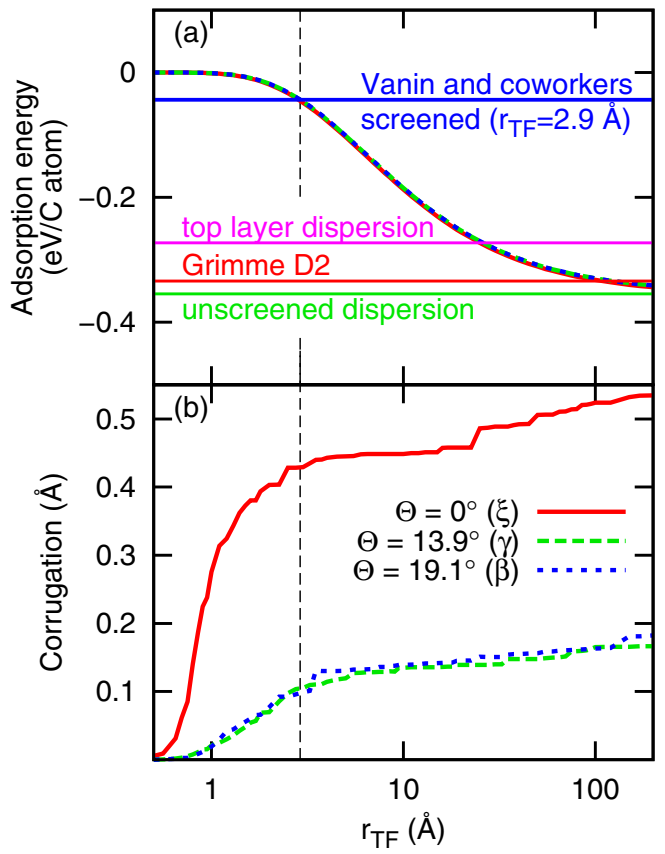

FIG. 11. (Color online) Variations of (a) adsorption energy and (b) corrugation of the graphene monolayer in epitaxial contact with $\mathrm{Pt}(111)$, as obtained from the implicit dispersion model as a function of the screening length $r_{\mathrm{TF}}$. The curves were obtained for various moiré structures corresponding to different in-plane angles $\Theta=0^{\circ}$ $(\xi), 13.9^{\circ}(\gamma)$, and $19.1^{\circ}(\beta)$ and based on local relaxations. In panel (a), the adsorption energies predicted by other dispersion models are also indicated as horizontal lines.

Alternatively, introducing a Thomas-Fermi screening factor as in Eq. (18) can achieve a similar effect, but to an even greater extent. Figure 11(a) shows the variations of the adsorption energy of the graphene layer in epitaxial contact with $\operatorname{Pt}(111)$, as a function of the screening length $r_{\mathrm{TF}}$ and for three different in-plane angles $\Theta$ corresponding to the structures denoted as $\xi, \gamma$, and $\beta$ in Table II. The damping of the global London attraction is particularly prominent, and the vdW-DFT reference value of $43 \mathrm{meV} /$ carbon atom is recovered approximately for $r_{\mathrm{TF}}=2.9 \AA$. The adsorption of epitaxial graphene is also found not to depend significantly on the type of moiré pattern, which is also true for the other dispersion models considered in this work (results not shown).

The natural corrugation of the graphene layer was evaluated from the locally relaxed structures and as a function of the screening length $r_{\mathrm{TF}}$. Its variations, shown in Fig. 11(b), are found to be correlated to those of the magnitude of the adsorption energy, which is the expected behavior since stronger binding also tends to increase the natural corrugation due to incommensurability and corrugate the carbon monolayer.

The interplay between corrugation and the strain exerted by the two materials in contact was further investigated by considering not only the magnitude $\Delta z$ of the graphene corrugation, but also the average separation $\delta z$ between the carbon sheet and the uppermost metal layer. Those properties were evaluated at $0 \mathrm{~K}$ from local minimizations of structures that were obtained via MD simulations at $300 \mathrm{~K}$. The variations of these geometric properties with increasing in-plane angle 


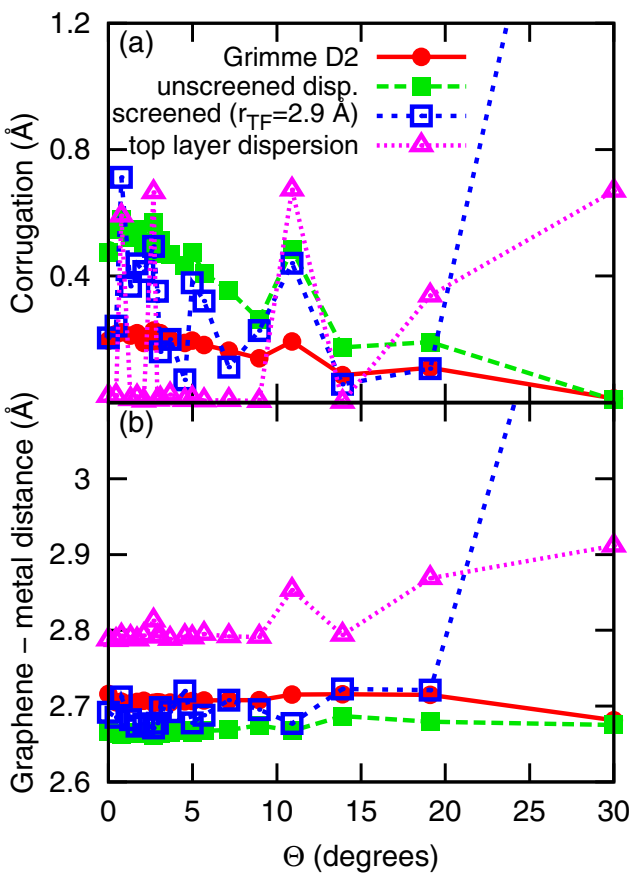

FIG. 12. (Color online) (a) Corrugation $\Delta z$ and (b) interlayer separation $\delta z$ of a graphene layer epitaxied on $\operatorname{Pt}(111)$ as a function of the in-plane angle $\Theta$, as obtained from different dispersion models at zero temperature. The values predicted by the model with screened dispersion forces lie beyond the respective upper values of the graphs at $\Theta=30^{\circ}$ (see text for discussion).

are represented in Fig. 12. As a general trend, corrugation decreases with increasing in-plane angle, which can be understood from the natural increase of the moiré periodicity with the in-plane angle. The average distance $\delta z$ is fairly independent of the in-plane angle and lies near the 2.8 - $\AA$ cutoff of the covalent part of the bond-order potential. Dispersion interactions affect the corrugation magnitude in a rather similar way as the adsorption energies in Fig. 10, where stronger dispersion leads to larger corrugations. This relation may be reversed, however, when the graphene layer experiences comparably high compressive strain, which is the case for the $\delta$ structure at $\Theta=10.9^{\circ}$, and especially in the $\xi$ moiré at $\Theta=$ $30^{\circ}$ where the unscreened models yield extreme values for the corrugation $(\Delta z=2.7 \AA)$ and separation $(\delta z=3.5 \AA)$ due to excessive lateral compression that cannot be compensated by the attraction to the metal. Limiting the dispersion forces from the uppermost metal layer attenuates this problem but does not solve it entirely. This effect is essentially geometric and arises due to slight squeezing or stretching of the graphene sheet necessary for matching the moiré within a common periodic simulation box also for the other moiré structures. Although we took care to perform all simulations with sufficiently large system sizes, size effects could still affect the corrugation of the graphene when it is highly subjected to compressive strain. Since graphene is a very stiff material [101], minor adjustments in the geometry produce relatively large effects especially when dispersion forces are weak or absent, as precisely occurs for the unscreened or top-layer dispersion models.

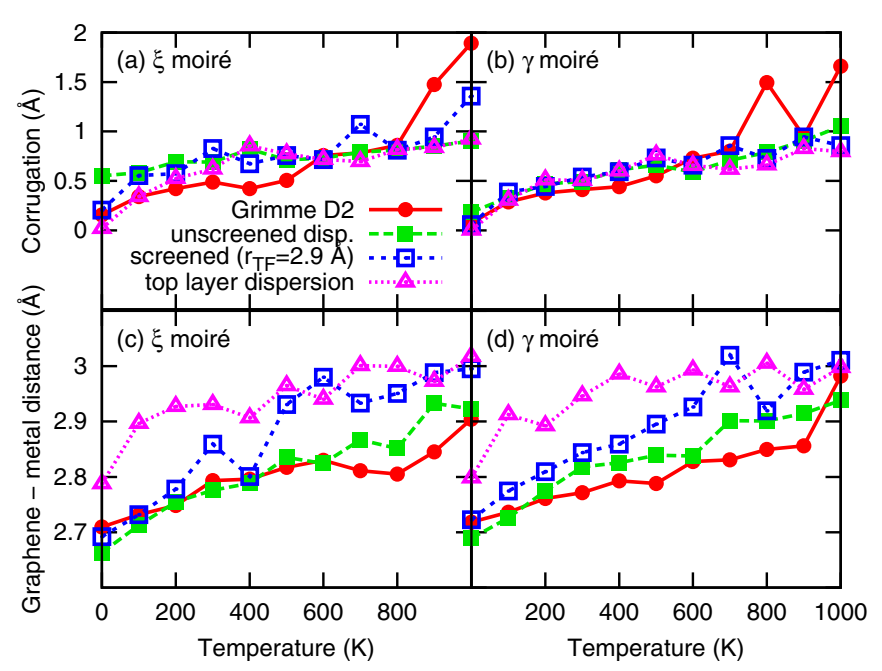

FIG. 13. (Color online) Thermally averaged corrugation $\Delta z$ (upper panels) and graphene-metal separation $\delta z$ (lower panels) of a graphene layer epitaxied on $\operatorname{Pt}(111)$ as a function of temperature, as obtained from different dispersion models for the $\xi$ moiré (in-plane angle $\Theta=0^{\circ}$, left panels) and the $\gamma$ moiré (in-plane angle $\Theta=13.9^{\circ}$, right panels).

Thermal effects were investigated for two specific moiré structures, namely, the $\xi$ and $\gamma$ patterns at $\Theta=0^{\circ}$ and $13.9^{\circ}$, respectively. MD simulations were carried out at increasing temperatures, and the average magnitudes of the corrugation and graphene-metal separation were both evaluated. They are reported in Fig. 13 for the four dispersion models. Once thermal effects are taken into account, the vibrational fluctuations make the graphene layer slightly shift away from the metal substrate for all models. According to Fig. 13, this shift amounts to approximately $0.1-0.3 \times 10^{-3} \AA \mathrm{K}^{-1}$. Thermal fluctuations also tend to increase the corrugation by about $0.3-1.4 \times 10^{-3} \AA \mathrm{K}^{-1}$ depending on the model. The four descriptions of dispersion forces agree well with each other for the $\xi$ moiré, but differ more sensitively for the other $\gamma$ structure, the top-layer dispersion model predicting some early increasing in both geometric properties but also some saturation already near room temperature.

For both values of the in-plane angles, the graphene layer experiences no compressive strain, and thermal effects are rather similar. Notable differences are found in moirés characterized by high compressive strain such as the $\delta$ or $\alpha$ structures, with stronger slopes and greater variations among models (data not shown). It should also be kept in mind that the simulation results presented in Fig. 13 were obtained at constant density. However, the two materials in contact have different thermal expansion coefficients and in practice simulations at finite pressure would be more appropriate. However, even then it would be rather difficult to find a common box size that is ideal for both materials, especially when their thermal expansion coefficients differ. While those refinements lie beyond the scope of this work, our results indicate how structural properties as basic as corrugation magnitude or interlayer separation are sensitive to the details of the interactions for such complex materials. 
Some comparison between the present results and available experimental measurements and electronic-structure calculations can also be attempted. Corrugations of epitaxial graphene accessed through STM height profiles have been reported to lie in the 0.3-0.8 $\AA$ range [88]. Those values are in good agreement with our calculations at $300 \mathrm{~K}$. Similar measurements carried by Land and co-workers [85] specifically for the $\xi$ structure have found that corrugation amounts to $0.8 \AA$, however, the authors indicate that part of this corrugation could be due to the tunneling current. In their density-functional study of the $\xi$ structure at $0 \mathrm{~K}$ (without dispersion corrections), Gao and co-workers [88] reported a buckling magnitude of $0.53 \AA$, which agrees with our calculation only in the extreme case of unscreened dispersion.

The graphene-metal separation has been experimentally determined by Sutter et al. [92] using LEED who found a value of about $3.3 \AA$. Similar values of $3.3 \AA$ [98] and $3.1 \AA$ [88] have been obtained in DFT calculations at $0 \mathrm{~K}$ and neglecting dispersion corrections, and surprisingly in their dispersion corrected DFT calculation Vanin and co-workers reported a significantly higher separation of $3.67 \AA$ [99], all these values again for the $\xi$ moiré structure. Those values are thus slightly higher than those obtained in the present model calculations, even at finite temperature, but it is clear that separations much larger than the $2.8-\AA$ cutoff of the covalent potential cannot be reached since the dispersion interaction is purely attractive.

The vibrational properties of epitaxial graphene can be straightforwardly evaluated from molecular dynamics simulations, allowing to demonstrate the potential of the present models to fully capture anharmonic properties as a function of temperature. The implicit dispersion model with screening length $r_{\mathrm{TF}}=2.9 \AA$ was employed for moiré structures of the $\xi$ and $\gamma$ types, and for comparison additional simulations were performed for freestanding graphene under the same density and lateral strain. From the 1-ns-long MD trajectories, the vibrational spectra were again obtained by Fourier transformation of the velocity time autocorrelation function. Similar to Fig. 8, one peak dominates the spectrum near $1600 \mathrm{~cm}^{-1}$ which we attribute to the $G$ band in the Raman spectrum of graphite [102]. This attribution is supported from normal mode analysis for increasingly strained structures, which indicate a dependence of the corresponding frequency of about $-71.2 \mathrm{~cm}^{-1}$ per percent of strain. Very similar values of $-63 \mathrm{~cm}^{-1} / \%$ [103] and $-70 \pm 3 \mathrm{~cm}^{-1} / \%$ [104] have been reported at $300 \mathrm{~K}$. Having assigned the calculated peak to the Raman $\mathrm{G}$ band, we have evaluated its robustness against finite-size effects by evaluating its variations with increasing size of the periodic cell in the simulation. Normal mode analysis indicates that convergence is reached already at $3 \times 3$ unit cells, as expected for such strong in-plane modes.

Because the moiré pattern sensitively depends on the lateral strain experienced by the epitaxial layer, it is expected that the vibrational signature of the $G$ band should depend on the in-plane angle. This speculation is confirmed from our numerical results, which have been represented in Fig. 14 as a function of temperature. For the three systems, the peak positions exhibit some nearly linear red-shifting with increasing temperature, which is the behavior known for pure graphite [105-107]. For pristine graphene, the variations show

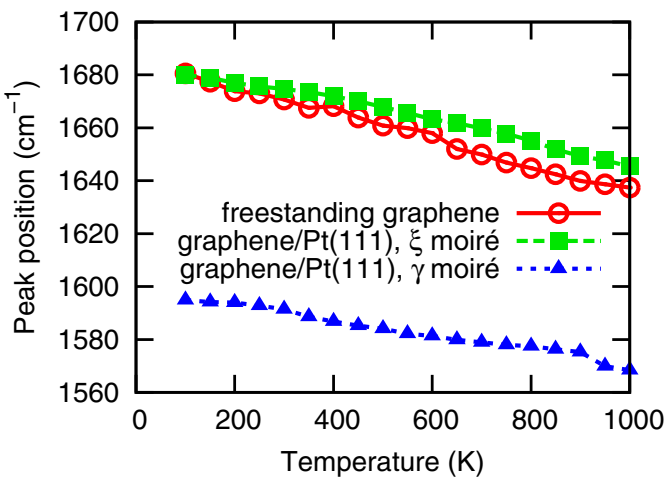

FIG. 14. (Color online) Position of the intense $G$ peak as a function of temperature, as obtained from MD simulations of freestanding graphene and epitaxial graphene on $\mathrm{Pt}(111)$ forming $\xi$ and $\gamma$ moiré patterns. The simulations of epitaxial graphene used the implicit dispersion correction model with a screening length of $r_{\mathrm{TF}}=2.9 \AA$.

a slope of approximately $-0.050 \mathrm{~cm}^{-1} \mathrm{~K}^{-1}$, in good agreement with Raman spectroscopy measurements [108-110].

In the case of epitaxial graphene, the $\mathrm{G}$ peak is not much altered in the $\xi$ moiré structure with no in-plane angle, whereas the $\gamma$ moiré exerts some rather high stretching strain leading to a significant red-shift of about 80 wave numbers already at low temperatures. As temperature is increased, the additional red-shift caused by anharmonicities is again manifested by nearly linear variations and slopes of $-0.039 \mathrm{~cm}^{-1} \mathrm{~K}^{-1}$ and $-0.028 \mathrm{~cm}^{-1} \mathrm{~K}^{-1}$ for the $\xi$ and $\gamma$ moirés, respectively. These results obtained for the $\xi$ moiré are in agreement with the only Raman measurements that we know of, namely, by Kang and co-workers [86] who reported some blue-shift of the $G$ band of epitaxial graphene on platinum relative to the bulk graphite due to compressive strain. In contrast to the $\xi$ structure which undergoes some compressive strain at finite temperature, strain in the $\gamma$ moiré is of the stretching type. Its manifestation as a red-shift in the vibrational spectrum is thus also consistent with the measurements of Kang et al. [86].

\section{B. Nanoparticles on moiré substrates}

The atomistic models developed so far are now applied to an even more complex situation of $\mathrm{Pt}$ adsorbates on epitaxial graphene on $\mathrm{Pt}(111)$. The two-layer $\mathrm{Pt}_{64}$ adsorbate was deposited onto the $\xi$ moiré structure initially at the fcc region and in the bridge epitaxial position and locally relaxed. We compare the predictions of the different dispersion correction models, namely, the fully explicit (Grimme D2) approach and the implicit model without any screening or with $r_{\mathrm{TF}}=2.9 \AA$. From the trajectories, the Lindemann indices of internal and adsorbate-substrate mobilities were calculated, as well as the adsorbate thickness used to monitor the possible structural transitions.

The variations of those indicators $\delta_{\text {intra }}, \delta_{\text {inter }}$, and $\sigma$ with increasing temperature, as obtained with the three models, have been represented in Figs. 15(a)-15(c). Interestingly, the fluxionality in the adsorbate as measured by $\delta_{\text {intra }}$ shows similar variations among the three models, with a rather flat behavior until $1000 \mathrm{~K}$ where sudden jumps occur. The 


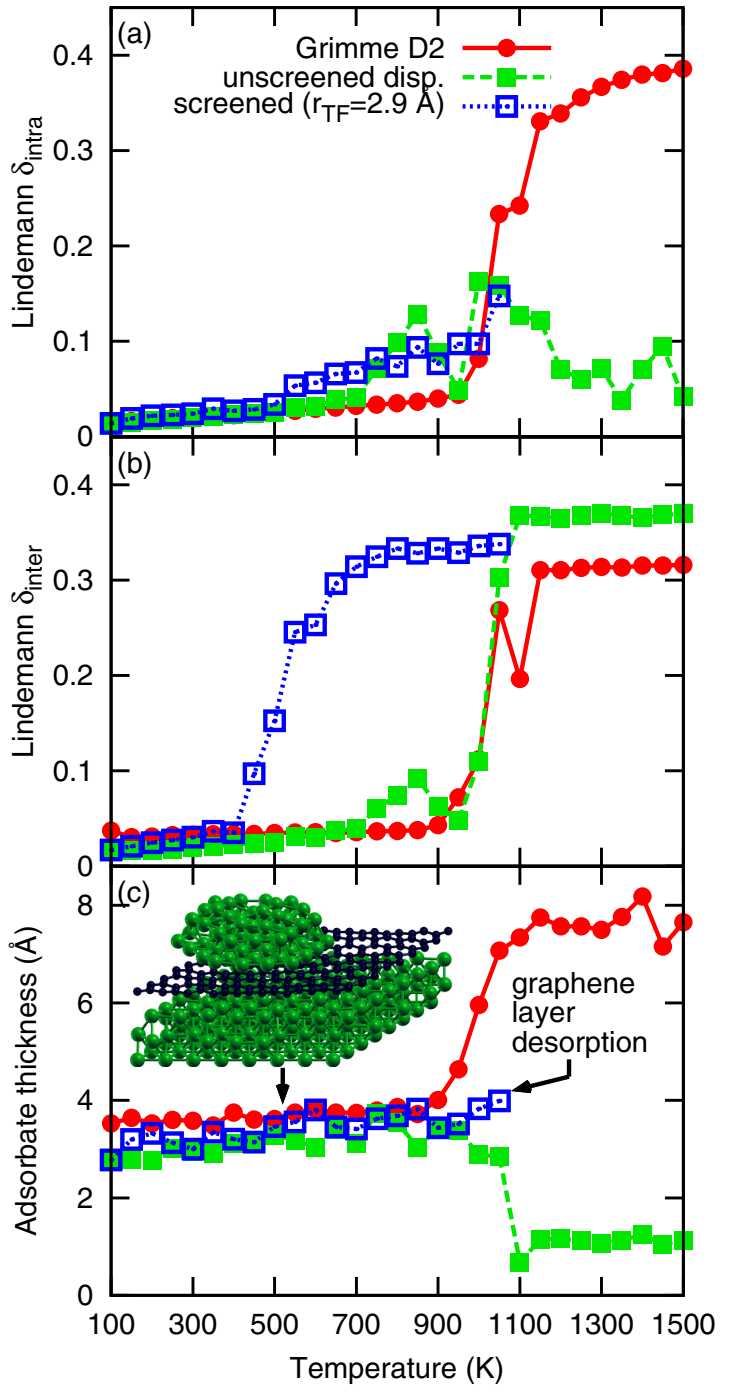

FIG. 15. (Color online) Lindemann indices of intra-adsorbate mobility and adsorbate-support and thickness of $\mathrm{Pt}_{64}$ on graphene/Pt(111) in the $\xi$ moiré structure as a function of temperature for different dispersion correction models.

adsorbate-substrate mobility index $\delta_{\text {inter }}$ is also similar between the explicit and unscreened implicit models, however, adsorbate mobility is clearly found with the screened implicit model already above $550 \mathrm{~K}$.

Inspection of the shape indicator $\sigma$ reveals notable differences that are reminiscent of similar conclusions obtained in the case of the graphite substrate in Fig. 6, with a tendency of the explicit model to produce more spherical shapes, and conversely flatter shapes with the unscreened implicit model. At temperatures exceeding $1100 \mathrm{~K}$, the adsorbate is highly disordered and no longer thermally stable. In addition, due to its low adsorption energy, the graphene sheet itself begins to thermally desorb above this temperature with the weakly binding screened dispersion model.

The diffusion dynamics can be probed from the meansquare displacement of the adsorbate more quantitatively than the index $\delta_{\text {inter }}$. Visual inspection indicates that even on this moiré substrate diffusion proceeds by a stick-slip mechanism with its Lévy flight properties. However, an additional mechanism is discernible in which the graphene layer slightly slides over the metal substrate (data not shown). This sliding process is found with all dispersion models, including the fully corrugated explicit treatment. Its contribution to the global diffusion is not obvious, nor its physical significance as such global motions could be markedly affected by size effects. In addition, the present approximation of a fixed density could also produce excessive strain in the substrate. Releasing this strain in constant-pressure simulations could well modify the diffusion behavior, although the problem of strain due to forcing the two subsystems in a common simulation box would remain.

\section{CONCLUSIONS}

London dispersion forces are an essential ingredient of sorption phenomena, and are expected to play a particularly important role for adsorbates on semi-infinite substrates. The contribution of long-range van der Waals attraction in density-functional theory is one of the most active topics in this field, and to a large extent this issue is also problematic in atomistic simulations relying on explicit potentials that model the metallic and covalent parts of chemical bonding. In this work, we have addressed this issue in the case of metallic nanoparticles deposited on pure carbonated substrates such as graphene or graphite, or epitaxial graphene on a bulk metal. Our approach is similar to the explicit Grimme scheme that considers atom-atom van der Waals interactions that add up to the covalent and metallic parts. However, in the same spirit as the Steele potential for substrate-atom interactions, we have also considered further the possibility of coarse graining this long-range contribution by integrating them rigorously over the layered semi-infinite substrate. This correction for a bulk substrate is especially relevant for the noncovalent contribution to binding because the bond-order potential ignores those contributions that lie beyond its cutoff. In the case of epitaxial graphene, the model was modified to account for possible screening effects that convey empirically the nonadditive nature of the van der Waals interaction over an electronically delocalized bulk medium.

The model was implemented and tested for platinum nanoparticles on carbonated substrates and epitaxial graphene on $\mathrm{Pt}(111)$, using the bond-order potential for the Pt-C system developed and parametrized by Albe and co-workers [47]. In the static limit for adsorption on graphite we find a good agreement between the explicit (Grimme D2) and coarse-grained implicit models for the adsorption energy of nanoparticles containing up to several thousand atoms, and comparison with available DFT data for the smaller adsorbates is also satisfactory. When used at finite temperature under molecular dynamics simulations, the explicit and coarse-grained models exhibit some dissimilarities, with a stronger tendency for wetting in the latter case and less diffusion over the substrate. This behavior was interpreted as due to a slightly stronger physisorption in the implicit model, whereas interactions are neglected in the explicit approach beyond the simulation cell. The resulting difference between the models, which amounts to approximately $20 \mathrm{meV} /$ atom, should obviously decrease in larger simulation supercells and bring the predictions of the explicit model closer to those of the coarse-grained model. 
The rate at which the two models converge would be worth investigating in the future, as it would also provide useful information for the DFT community about the importance of those corrections that are no longer minor once integrated over the (true) infinite substrate. Diffusion mechanisms on the graphite substrate were found to proceed via truncated Lévy flights, as previously identified in the literature [79-81].

The model was also extended to describe epitaxial graphene on fcc platinum, and notably the moiré patterns that such two lattices produce when in contact with one another due to their incommensurability. In the case where the moire is altered by some in-plane angle, the geometrical and finite-temperature properties of those patterned substrates were found to depend nontrivially on this angle. The corrugation of the graphene layer and its separation to the uppermost metal layer were found to be comparable to existing reference data, provided that the dispersion interactions are properly screened: An optimal screening length was evaluated to be as low as $2.9 \AA$. The vibrational spectra in the region of the Raman $\mathrm{G}$ peak of graphite, as obtained with a complete account of anharmonicities through the velocity time correlation function, were found to display also notable shifts and variations with temperature and the in-plane angle. Those predictions, which should be amenable to comparison with experiment, highlight the importance of compressive or stretching strain exerted at the metal/carbon contact in the moiré pattern. The dynamics of Pt adsorbates on epitaxial graphene was also investigated and shown to be similar to that on graphite, again with sensible dynamical differences arising from the nature of the dispersion correction employed in the modeling. In particular, only the most realistic dispersion model that accounts for nonadditive screening effects suggests that the graphene layer with the adsorbates should thermally desorb above $1000 \mathrm{~K}$, the adsorbates being excessively pulled to the substrate in absence of screening.

Some limitations in our modeling could be noted in the case of graphene, where for large adsorbed nanoparticles the deformation of the carbon monolayer compromises the planar approximation employed in the integrated dispersion correction. Although large nanoparticles deposited on pristine graphene may be more of academic interest, one possibly general way of addressing this issue could be to use the implicit model only beyond the simulation cell box, and keeping an atomistic description within. Under such hybrid models, and as with other boundary problems in multiscale approaches, the transition between the atomistic and coarsegrained contributions should be carefully chosen. In the case of bulk substrates, the implicit model seems to be generally valid, although ultimately the accuracy of its predictions should be evaluated against experiment rather than electronicstructure calculations (especially those lacking screening), the underlying model being semiempirical instead of $a b$ initio based.

The physical properties of Pt nanoparticles on carbonated substrates were found here to depend quite sensitively on dispersion forces. Those conclusions may convey to other metals that are also weakly bound to carbon such as iridium [4], but we do not expect such a great role in the case of other transition metals such as nickel [111] or ruthenium [112], for which sorption is more of the chemical type. Extension of the present model to those metals is currently under way.

It would also be worth extending the Pt-C model to treat additional hydrogen atoms, which would imply some further parametrization for terms involving hydrogen and platinum atoms (the Brenner model can be readily used for $\mathrm{C}-\mathrm{H}$ atoms). Such an extension would pave the way to model hydrocarbon molecules on metal surfaces, for which the concerted effects of covalent and van der Waals forces have been discussed at length [113], including the specific issue of screening. It could then become necessary to account for charge transfer and polarization effects more explicitly (at least those leading to the multipolar nature of the adsorbed molecules), which would also imply considering the possible effects of image charges.

One immediate application of the coarse-grained model would be the determination of optimal shapes for nanoparticles containing typically a hundred atoms and placed on the deformable carbonated substrates discussed in this work. Global optimization would be useful notably for particles on epitaxial graphene, owing to promises in magnetic storage of such materials $[4,114,115]$. One other important application of the model could be to treat larger 2D assemblies of many adsorbates. At equilibrium, the stable patterns adopted by the assemblies should not only depend on the substrate (and the possible presence of a moiré nanomesh) but also on the adsorbate itself, as well as other external factors such as diffusion, adsorption, or thermal desorption [116]. Long-range forces could also contribute to stabilizing the assemblies and should be incorporated in the model as well. Assemblies of nanoparticles on surfaces could be described at a fully atomistic level, but also in a more global fashion by extracting the effective interactions between the nanoparticles at finite temperature and plugging those into a coarse-grained model whose evolution is ruled, e.g., by dissipative particle dynamics [117].

\section{ACKNOWLEDGMENTS}

We gratefully acknowledge support from the Pôle Scientifique de Modélisation Numérique (PSMN) at ENS Lyon. The authors thank C. Bichara for fruitful discussions and useful suggestions. Funding from Agence Nationale de La Recherche (ANR Project No. NMGEM 16-339233) is also acknowledged.
[1] D. L. Feldheim and C. A. Foss Jr., Metal Nanoparticles: Synthesis, Characterization and Applications (Marcel Dekker, New York, 2002).

[2] I. Fernandez-Torrente, S. Monturet, K. J. Franke, J. Fraxedas, N. Lorente, and J. I. Pascual, Long-range repulsive interaction between molecules on a metal surface induced by charge transfer, Phys. Rev. Lett. 99, 176103 (2007).

[3] S. Kinge, M. Crego-Calama, and D. N. Reinhoudt, Selfassembling nanoparticles at surfaces and interfaces, Chem. Phys. Chem. 9, 20 (2008). 
[4] A. T. N'Diaye, S. Bleikamp, P. J. Feibelman, and T. Michely, Two-dimensional Ir cluster lattice on a graphene moiré on Ir(111), Phys. Rev. Lett. 97, 215501 (2006).

[5] L. Bardotti, F. Tournus, P. Mélinon, M. Pellarin, and M. Broyer, Mass-selected clusters deposited on graphite: Spontaneous organization controlled by cluster surface reaction, Phys. Rev. B 83, 035425 (2011).

[6] J. Coraux, A. T. N'Diaye, N. Rougemaille, C. Vo-Van, A. Kimouche, H.-X. Yang, M. Chshiev, N. Bendiab, O. Fruchart, and A. K. Schmid, Air-protected epitaxial graphene/ferromagnet hybrids prepared by chemical vapor deposition and intercalation, J. Phys. Chem. Lett. 3, 2059 (2012).

[7] A. Cavallin, M. Pozzo, C. Africh, A. Baraldi, E. Vesselli, C. Dri, G. Comelli, R. Larciprete, P. Lacovig, S. Lizzit, and D. Alfè, Local electronic structure and density of edge and facet atoms at Rh nanoclusters self-assembled on a graphene template, ACS Nano 6, 3034 (2012).

[8] K. Yamamoto, T. Imaoka, W.-J. Chun, O. Enoki, H. Katoh, M. Takenaga, and A. Sonoi, Size-specific catalytic activity of platinum clusters enhances oxygen reduction reactions, Nat. Chem. 1, 397 (2009).

[9] R. Siburian, T. Kondo, and J. Nakamura, Size control to a sub-nanometer scale in platinum catalysts on graphene, J. Phys. Chem. C 117, 3635 (2013).

[10] Y. Shen, Z. Zhang, K. Xiao, and J. Xi, Electrocatalytic activity of $\mathrm{Pt}$ subnano/nanoclusters stabilized by pristine graphene nanosheets, Phys. Chem. Chem. Phys. 16, 21609 (2014).

[11] Y. Si and E. T. Samulski, Exfoliated graphene separated by platinum nanoparticles, Chem. Mater. 20, 6792 (2008).

[12] L. Wang and R. T. Yang, Hydrogen storage properties of carbons doped with ruthenium, platinum, and nickel nanoparticles, J. Phys. Chem. C 112, 12486 (2008).

[13] D.-H. Lim and J. Wilcox, Mechanisms of the oxygen reduction reaction on defective graphene-supported $\mathrm{Pt}$ nanoparticles from first-principles, J. Phys. Chem. C 116, 3653 (2012).

[14] S. Hu, M. Lozada-Hidalgo, F. C. Wang, A. Mishchenko, F. Schedin, R. R. Nair, E. W. Hill, D. W. Boukhvalov, M. I. Katsnelson, R. A. W. Dryfe, I. V. Grigorieva, H. A. Wu, and A. K. Geim, Proton transport through one-atom-thick crystals, Nature (London) 516, 227 (2014).

[15] P. Sony, P. Puschnig, D. Nabok, and C. Ambrosch-Draxl, Importance of van der Waals interaction for organic moleculemetal junctions: Adsorption of thiophene on $\mathrm{Cu}(110)$ as a prototype, Phys. Rev. Lett. 99, 176401 (2007).

[16] G. Mercurio, E. R. McNellis, I. Martin, S. Hagen, F. Leyssner, S. Soubatch, J. Meyer, M. Wolf, P. Tegeder, F. S. Tautz, and K. Reuter, Structure and energetics of azobenzene on $\mathrm{Ag}(111)$ : Benchmarking semiempirical dispersion correction approaches, Phys. Rev. Lett. 104, 036102 (2010).

[17] N. Atodiresei, V. Caciuc, P. Lazić, and S. Blügel, Chemical versus van der Waals interaction: The role of the heteroatom in the flat absorption of aromatic molecules $\mathrm{C}_{6} \mathrm{H}_{6}, \mathrm{C}_{5} \mathrm{NH}_{5}$, and $\mathrm{C}_{4} \mathrm{~N}_{2} \mathrm{H}_{4}$ on the $\mathrm{Cu}(110)$ surface, Phys. Rev. Lett. 102, 136809 (2009).

[18] J. Brede, N. Atodiresei, S. Kuck, P. Lazić, V. Caciuc, Y. Morikawa, G. Hoffmann, S. Blügel, and R. Wiesendanger, Spin- and energy-dependent tunneling through a single molecule with intramolecular spatial resolution, Phys. Rev. Lett. 105, 047204 (2010).
[19] E. R. McNellis, C. Bronner, J. Meyer, M. Weinelt, P. Tegeder, and K. Reuter, Azobenzene versus 3,3',5,5'-tetra-tert-butylazobenzene (TBA) at $\mathrm{Au}(111)$ : Characterizing the role of spacer groups, Phys. Chem. Chem. Phys. 12, 6404 (2010).

[20] M. Mura, A. Gulans, T. Thonhauser, and L. Kantorovich, Role of van der Waals interaction in forming molecule-metal junctions: Flat organic molecules on the $\mathrm{Au}(111)$ surface, Phys. Chem. Chem. Phys. 12, 4759 (2010).

[21] A. Tkatchenko, Current understanding of van der Waals effects in realistic materials, Adv. Funct. Mater. 25, 2054 (2015).

[22] D. Stradi, S. Barja, C. Díaz, M. Garnica, B. Borca, J. J. Hinarejos, D. Sánchez-Portal, M. Alcamí, A. Arnau, A. L. Vázquez de Parga, R. Miranda, and F. Martín, Role of dispersion forces in the structure of graphene monolayers on Ru surfaces, Phys. Rev. Lett. 106, 186102 (2011).

[23] P. Janthon, F. Viñes, S. M. Kozlov, J. Limtrakul, and F. Illas, Theoretical assessment of graphene-metal contacts, J. Chem. Phys. 138, 244701 (2013).

[24] M. P. Andersson, Density functional theory with modified dispersion correction for metals applied to self-assembled monolayers of thiols on $\mathrm{Au}(111)$, J. Theor. Chem. 2013, 327839 (2013).

[25] S. Grimme, Density functional theory with London dispersion corrections, Wiley Interdiscip. Rev.: Comput. Mol. Sci. 1, 211 (2011).

[26] M. Dion, H. Rydberg, E. Schröder, D. C. Langreth, and B. I. Lundqvist, Van der Waals density functional for general geometries, Phys. Rev. Lett. 92, 246401 (2004).

[27] K. Lee, É. D. Murray, L. Kong, B. I. Lundqvist, and D. C. Langreth, Higher-accuracy van der Waals density functional, Phys. Rev. B 82, 081101 (2010).

[28] Y. Zhao and D. G. Truhlar, Density functionals with broad applicability in chemistry, Acc. Chem. Res. 41, 157 (2008).

[29] L. Ferrighi, Y.-X. Pan, H. Grönbeck, and B. Hammer, Study of alkylthiolate self-assembled monolayers on $\mathrm{Au}(111)$ using a semilocal meta-GGA density functional, J. Phys. Chem. C 116, 7374 (2012).

[30] S. Grimme, Accurate description of van der Waals complexes by density functional theory including empirical corrections, J. Comput. Chem. 25, 1463 (2004).

[31] S. Grimme, Semiempirical GGA-type density functional constructed with a long-range dispersion correction, J. Comput. Chem. 27, 1787 (2006).

[32] S. Grimme, J. Antony, S. Ehrlich, and H. Krieg, A consistent and accurate ab initio parametrization of density functional dispersion correction (DFT-D) for the 94 elements $\mathrm{H}-\mathrm{Pu}, \mathrm{J}$. Chem. Phys. 132, 154104 (2010).

[33] S. Grimme, S. Ehrlich, and L. Goerigk, Effect of the damping function in dispersion corrected density functional theory, $\mathrm{J}$. Comput. Chem. 32, 1456 (2011).

[34] P. Jurečka, J. Černý, P. Hobza, and D. R. Salahub, Density functional theory augmented with an empirical dispersion term. Interaction energies and geometries of 80 noncovalent complexes compared with ab initio quantum mechanics calculations, J. Comput. Chem. 28, 555 (2007).

[35] P. L. Silvestrelli, Van der Waals interactions in DFT made easy by Wannier functions, Phys. Rev. Lett. 100, 053002 (2008).

[36] A. Tkatchenko and M. Scheffler, Accurate molecular van der Waals interactions from ground-state electron density and freeatom reference data, Phys. Rev. Lett. 102, 073005 (2009). 
[37] R. Podgornik, R. H. French, and V. A. Parsegian, Nonadditivity in van der Waals interactions within multilayers, J. Chem. Phys. 124, 044709 (2006).

[38] B. E. Sernelius and C. E. Román-Velázquez, Beyond the simple proximity force approximation: Geometrical effects on the nonretarded Casimir interaction, Phys. Rev. A 78, 032111 (2008).

[39] J. Sarabadani, A. Naji, R. Asgari, and R. Podgornik, Manybody effects in the van der Waals-Casimir interaction between graphene layers, Phys. Rev. B 84, 155407 (2011).

[40] C. Wagner, N. Fournier, V. G. Ruiz, C. Li, K. Müllen, M. Rohlfing, A. Tkatchenko, R. Temirov, and F. S. Tautz, Nonadditivity of molecule-surface van der Waals potentials from force measurements, Nat. Commun. 5, 5568 (2014).

[41] B. Chilukuri, U. Mazur, and K. W. Hipps, Effect of dispersion on surface interactions of cobalt(II) octaethylporphyrin monolayer on $\mathrm{Au}(111)$ and $\mathrm{HOPG}(0001)$ substrates: A comparative first principles study, Phys. Chem. Chem. Phys. 16, 14096 (2014).

[42] R. A. Boto, J. Contreras-García, and M. Calatayud, The role of dispersion forces in metal-supported self-assembled monolayers, Comput. Theor. Chem. 1053, 322 (2015).

[43] G. Ramos-Sanchez and P. B. Balbuena, Interactions of platinum clusters with a graphite substrate, Phys. Chem. Chem. Phys. 15, 11950 (2013).

[44] K. Saitoh and H. Hayakawa, Simulation of depositions of a Lennard-Jones cluster on a crystalline surface, Prog. Theor. Phys. 122, 1081 (2009).

[45] W. A. Steele, The physical interaction of gases with crystalline solids: I. Gas-solid energies and properties of isolated adsorbed atoms, Surf. Sci. 36, 317 (1973).

[46] D. W. Brenner, Empirical potential for hydrocarbons for use in simulating the chemical vapor deposition of diamond films, Phys. Rev. B 42, 9458 (1990).

[47] K. Albe, K. Nordlund, and R. S. Averback, Modeling the metalsemiconductor interaction: Analytical bond-order potential for platinum-carbon, Phys. Rev. B 65, 195124 (2002).

[48] G. C. Abell, Empirical chemical pseudopotential theory of molecular and metallic bonding, Phys. Rev. B 31, 6184 (1985).

[49] J. Tersoff, New empirical model for the structural properties of silicon, Phys. Rev. Lett. 56, 632 (1986).

[50] D. W. Brenner, Relationship between the embedded-atom method and Tersoff potentials, Phys. Rev. Lett. 63, 1022 (1989).

[51] M. S. Daw and M. I. Baskes, Semiempirical, quantum mechanical calculation of hydrogen embrittlement in metals, Phys. Rev. Lett. 50, 1285 (1983).

[52] M. S. Daw and M. I. Baskes, Embedded-atom method: Derivation and application to impurities, surfaces, and other defects in metals, Phys. Rev. B 29, 6443 (1984).

[53] P. Pyykkö and M. Atsumi, Molecular double-bond covalent radii for elements Li-E112, Chem.-Eur. J. 15, 12770 (2009).

[54] G. D. Förster, Y. Magnin, F. Rabilloud, and F. Calvo, Effective embedded-atom potential for metallic adsorbates on crystalline surfaces, Modell. Simul. Mater. Sci. Eng. 22, 035015 (2014).

[55] J. Che, T. Çăğın, and W. A. Goddard III, Generalized extended empirical bond-order dependent force fields including nonbond interactions, Theor. Chem. Acc. 102, 346 (1999).

[56] T. Gould, S. Lebègue, and J. F. Dobson, Dispersion corrections in graphenic systems: A simple and effective model of binding, J. Phys.: Condens. Matter 25, 445010 (2013).
[57] B. G. Janesko, T. M. Henderson, and G. E. Scuseria, Screened hybrid density functionals for solid-state chemistry and physics, Phys. Chem. Chem. Phys. 11, 443 (2009).

[58] P. L. Silvestrelli, A. Ambrosetti, S. Grubisice, and F. Ancilotto, Adsorption of rare-gas atoms on $\mathrm{Cu}(111)$ and $\mathrm{Pb}(111)$ surfaces by van der Waals corrected density functional theory, Phys. Rev. B 85, 165405 (2012).

[59] P. L. Silvestrelli and A. Ambrosetti, Inclusion of screening effects in the van der Waals corrected DFT simulation of adsorption processes on metal surfaces, Phys. Rev. B 87, 075401 (2013).

[60] P. L. Silvestrelli and A. Ambrosetti, Including screening in van der Waals corrected density functional theory calculations: The case of atoms and small molecules physisorbed on graphene, J. Chem. Phys. 140, 124107 (2014).

[61] E. W. Weisstein, CRC Concise Encyclopedia of Mathematics, 2nd ed. (CRC Press, Boca Raton, FL, 2002).

[62] A. I. Frenkel, C. W. Hills, and R. G. Nuzzo, A view from the inside: Complexity in the atomic scale ordering of supported metal nanoparticles, J. Phys. Chem. B 105, 12689 (2001).

[63] D. Franz, S. Runte, C. Busse, S. Schumacher, T. Gerber, T. Michely, M. Mantilla, V. Kilic, J. Zegenhagen, and A. Stierle, Atomic structure and crystalline order of graphenesupported Ir nanoparticle lattices, Phys. Rev. Lett. 110, 065503 (2013).

[64] M. Bassiouk, E. Álvarez Zauco, and V. A. Basiuk, Theoretical analysis of the effect of surface defects on porphyrin adsorption and self-assembly on graphite, J. Comput. Theor. Nanosci. 9, 532 (2012).

[65] A. Perez, L. Bardotti, B. Prevel, P. Jensen, M. Treilleux, P. Mélinon, J. Gierak, G. Faini, and D. Mailly, Quantumdot systems prepared by 2D organization of nanoclusters preformed in the gas phase on functionalized substrates, New J. Phys. 4, 76 (2002).

[66] X. Tang, X. Li, Y. Wang, K. Wepasnick, A. Lim, D. H. Fairbrother, K. H. Bowen, T. Mangler, S. Noessner, C. Wolke, M. Grossmann, A. Koop, G. Gantefoer, B. Kiran, and A. K. Kandalam, Size selected clusters on surfaces, J. Phys.: Conf. Ser. 438, 012005 (2013).

[67] X. Li, K. Wepasnick, X. Tang, D. H. Fairbrother, K. H. Bowen, A. Dollinger, C. H. Strobel, J. Huber, T. Mangler, Y. Luo, S. Proch, and G. Gantefoer, A new nanomaterial synthesized from size-selected, ligand-free metal clusters, J. Appl. Phys. 115, 104304 (2014).

[68] S. Linas, F. Jean, T. Zhou, C. Albin, G. Renaud, L. Bardotti, and F. Tournus, Moiré induced organization of size-selected Pt clusters soft landed on epitaxial graphene, arXiv:1412.0487 [Sci. Rep. (to be published)].

[69] F. Baletto and R. Ferrando, Structural properties of nanoclusters: Energetic, thermodynamic, and kinetic effects, Rev. Mod. Phys. 77, 371 (2005).

[70] M. N. Groves, C. Malardier-Jugroot, and M. Jugroot, Improving platinum catalyst durability with a doped graphene support, J. Phys. Chem. C 116, 10548 (2012).

[71] G. Kim, Y. Kawazoe, and K.-R. Lee, Controlled catalytic properties of platinum clusters on strained graphene, J. Phys. Chem. Lett. 3, 1989 (2012).

[72] I. Fampiou and A. Ramasubramaniam, Binding of Pt nanoclusters to point defects in graphene: Adsorption, morphology, and electronic structure, J. Phys. Chem. C 116, 6543 (2012). 
[73] K.-J. Kong, Y. Choi, B.-H. Ryu, J.-O. Lee, and H. Chang, Investigation of metal/carbon-related materials for fuel cell applications by electronic structure calculations, J. Mater. Sci. Eng. C 26, 1207 (2006).

[74] K. Okazaki-Maeda, Y. Morikawa, S. Tanaka, and M. Kohyama, Structures of Pt clusters on graphene by first-principles calculations, Surf. Sci. 604, 144 (2010).

[75] A. Rochefort, D.-Q. Yang, and E. Sacher, Stabilization of platinum nanoparticles on graphene by non-invasive functionalization, Carbon 47, 2233 (2009).

[76] Y. Okamoto, Density-functional calculations of icosahedral $\mathrm{M}_{13}(\mathrm{M}=\mathrm{Pt}$ and $\mathrm{Au})$ clusters on graphene sheets and flakes, Chem. Phys. Lett. 420, 382 (2006).

[77] Q. Qi, H. Liu, W. Feng, H. Tian, H. Xu, and X. Huang, Theoretical investigation on the interaction of subnano platinum clusters with graphene using DFT methods, Comput. Mater. Sci. 96, 268 (2015).

[78] R. N. Mantegna and H. E. Stanley, Stochastic process with ultraslow convergence to a Gaussian: The truncated Lévy flight, Phys. Rev. Lett. 73, 2946 (1994).

[79] W. D. Luedtke and U. Landman, Slip diffusion and Lévy flights of an adsorbed gold nanocluster, Phys. Rev. Lett. 82, 3835 (1999).

[80] Y. Maruyama and J. Murakami, Truncated Lévy walk of a nanocluster bound weakly to an atomically flat surface: Crossover from superdiffusion to normal diffusion, Phys. Rev. B 67, 085406 (2003).

[81] Y. Maruyama, Temperature dependence of Lévy-type stick-slip diffusion of a gold nanocluster on graphite, Phys. Rev. B 69, 245408 (2004).

[82] O. Stein, J. Ankri, and M. Asscher, Surface diffusion of gold nanoclusters on $\mathrm{Ru}(0001)$ : Effects of cluster size, surface defects and adsorbed oxygen atoms, Phys. Chem. Chem. Phys. 15, 13506 (2013).

[83] H. Tetlow, J. Posthuma de Boer, I. J. Ford, D. D. Vvedensky, J. Coraux, and L. Kantorovich, Growth of epitaxial graphene: Theory and experiment, Phys. Rep. 542, 195 (2014).

[84] M. Enachescu, D. Schleef, D. F. Ogletree, and M. Salmeron, Integration of point-contact microscopy and atomic-force microscopy: Application to characterization of graphite/Pt(111), Phys. Rev. B 60, 16913 (1999).

[85] T. A. Land, T. Michely, R. J. Behm, J. C. Hemminger, and G. Comsa, STM investigation of single layer graphite structures produced on $\mathrm{Pt}(111)$ by hydrocarbon decomposition, Surf. Sci. 264, 261 (1992).

[86] B. J. Kang, J. H. Mun, C. Y. Hwang, and B. J. Cho, Monolayer graphene growth on sputtered thin film platinum, J. Appl. Phys. 106, 104309 (2009).

[87] M. Sasaki, Y. Yamada, Y. Ogiwara, S. Yagyu, and S. Yamamoto, Moiré contrast in the local tunneling barrier height images of monolayer graphite on Pt(111), Phys. Rev. B 61, 15653 (2000).

[88] M. Gao, Y. Pan, L. Huang, H. Hu, L. Z. Zhang, H. M. Guo, S. X. Du, and H.-J. Gao, Epitaxial growth and structural property of graphene on $\mathrm{Pt}(111)$, Appl. Phys. Lett. 98, 033101 (2011).

[89] G. W. Cushing, V. Johánek, J. K. Navin, and I. Harrison, Graphene growth on $\mathrm{Pt}(111)$ by ethylene chemical vapor deposition at surface temperatures near $1000 \mathrm{~K}$, J. Phys. Chem. C 119, 4759 (2015).
[90] H. Ueta, M. Saida, C. Nakai, Y. Yamada, M. Sasaki, and S. Yamamoto, Highly oriented monolayer graphite formation on $\mathrm{Pt}(111)$ by a supersonic methane beam, Surf. Sci. 560, 183 (2004).

[91] P. Merino, M. Švec, A. L. Pinardi, G. Otero, and J. A. Martín-Gago, Strain-driven moiré superstructures of epitaxial graphene on transition metal surfaces, ACS Nano 5, 5627 (2011).

[92] P. Sutter, J. T. Sadowski, and E. Sutter, Graphene on Pt(111): Growth and substrate interaction, Phys. Rev. B 80, 245411 (2009).

[93] S. Grandthyll, S. Gsell, M. Weinl, M. Schreck, S. Hüfner, and F. Müller, Epitaxial growth of graphene on transition metal surfaces: Chemical vapor deposition versus liquid phase deposition, J. Phys.: Condens. Matter 24, 314204 (2012).

[94] J. Wintterlin and M.-L. Bocquet, Graphene on metal surfaces, Surf. Sci. 603, 1841 (2009).

[95] E. Sutter, P. Albrecht, B. Wang, M.-L. Bocquet, L. Wu, Y. Zhu, and P. Sutter, Arrays of Ru nanoclusters with narrow size distribution templated by monolayer graphene on Ru, Surf. Sci. 605, 1676 (2011).

[96] K. Hermann, Periodic overlayers and moiré patterns: Theoretical studies of geometric properties, J. Phys.: Condens. Matter 24, 314210 (2012).

[97] P.Zeller and S. Günther, What are the possible moiré patterns of graphene on hexagonally packed surfaces? Universal solution for hexagonal coincidence lattices, derived by a geometric construction, New J. Phys. 16, 083028 (2014).

[98] P. A. Khomyakov, G. Giovannetti, P. C. Rusu, G. Brocks, J. van den Brink, and P. J. Kelly, First-principles study of the interaction and charge transfer between graphene and metals, Phys. Rev. B 79, 195425 (2009).

[99] M. Vanin, J. J. Mortensen, A. K. Kelkkanen, J. M. GarciaLastra, K. S. Thygesen, and K. W. Jacobsen, Graphene on metals: A van der Waals density functional study, Phys. Rev. B 81, 081408 (2010).

[100] F. Hanke, M. S. Dyer, J. Björk, and M. Persson, Structure and stability of weakly chemisorbed ethene adsorbed on lowindex Cu surfaces: Performance of density functionals with van der Waals interactions, J. Phys.: Condens. Matter 24, 424217 (2012).

[101] G. Tsoukleri, J. Parthenios, K. Papagelis, R. Jalil, A. C. Ferrari, A. K. Geim, K. S. Novoselov, and C. Galiotis, Subjecting a graphene monolayer to tension and compression, Small $\mathbf{5}$, 2397 (2009).

[102] F. Tuinstra and J. L. Koenig, Raman spectrum of graphite, J. Chem. Phys. 53, 1126 (1970).

[103] T. M. G. Mohiuddin, A. Lombardo, R. R. Nair, A. Bonetti, G. Savini, R. Jalil, N. Bonini, D. M. Basko, C. Galiotis, N. Marzari, K. S. Novoselov, A. K. Geim, and A. C. Ferrari, Uniaxial strain in graphene by Raman spectroscopy: $G$ peak splitting, Grüneisen parameters, and sample orientation, Phys. Rev. B 79, 205433 (2009).

[104] D. Yoon, Y.-W. Son, and H. Cheong, Strain-dependent splitting of the double-resonance Raman scattering band in graphene, Phys. Rev. Lett. 106, 155502 (2011).

[105] K. Hiroyuki, T. Ikari, W. Masao, T. Kazuya, K. Nao, I. Keisuke, and W. Hideki, Proper understanding of down-shifted Raman spectra of natural graphite: Direct estimation of laser-induced 
rise in sample temperature, Geochim. Cosmochim. Acta 58, 3527 (1994).

[106] P. H. Tan, Y. M. Deng, Q. Zhao, and W. C. Cheng, The intrinsic temperature effect of the Raman spectra of graphite, Appl. Phys. Lett. 74, 1818 (1999).

[107] N. Bonini, M. Lazzeri, N. Marzari, and F. Mauri, Phonon anharmonicities in graphite and graphene, Phys. Rev. Lett. 99, 176802 (2007)

[108] I. Calizo, A. A. Balandin, W. Bao, F. Miao, and C. N. Lau, Temperature dependence of the Raman spectra of graphene and graphene multilayers, Nano Lett. 7, 2645 (2007).

[109] D. Yoon, Y.-W. Son, and H. Cheong, Negative thermal expansion coefficient of graphene measured by Raman spectroscopy, Nano Lett. 11, 3227 (2011).

[110] S. Linas, Y. Magnin, B. Poinsot, O. Boisron, G. D. Förster, V. Martinez, R. Fulcrand, F. Tournus, V. Dupuis, F. Rabilloud, L. Bardotti, Z. Han, D. Kalita, V. Bouchiat, and F. Calvo, Interplay between Raman shift and thermal expansion in graphene: Temperature-dependent measurements and analysis of substrate corrections, Phys. Rev. B 91, 075426 (2015).

[111] W. Zhao, S. M. Kozlov, O. Höfert, K. Gotterbarm, M. P. A. Lorenz, F. Viñes, C. Papp, A. Görling, and H.-P. Steinrück,
Graphene on Ni(111): Coexistence of different surface structures, J. Phys. Chem. Lett. 2, 759 (2011).

[112] W. Moritz, B. Wang, M.-L. Bocquet, T. Brugger, T. Greber, J. Wintterlin, and S. Günther, Structure determination of the coincidence phase of graphene on $\mathrm{Ru}(0001)$, Phys. Rev. Lett. 104, 136102 (2010).

[113] W. Liu, J. Carrasco, B. Santra, A. Michaelides, M. Scheffler, and A. Tkatchenko, Benzene adsorbed on metals: Concerted effect of covalency and van der Waals bonding, Phys. Rev. B 86, 245405 (2012).

[114] S. P. Gubin, Y. I. Spichkin, G. Y. Yurkov, and A. M. Tishin, Nanomaterial for high-density magnetic data storage, Russ. J. Inorg. Chem. 47, S32 (2002).

[115] K.-L. Wu, X.-Z. Li, X.-W. Wei, T.-H. Ding, M. Jiang, W.-J. Zhang, and Y. Ye, Controllable synthesis and property of graphene-based magnetic metal nanostructures, Solid State Sci. 38, 90 (2014).

[116] P. Jensen, Growth of nanostructures by cluster deposition: Experiments and simple models, Rev. Mod. Phys. 71, 1695 (1999).

[117] P. J. Hoogerbrugge and J. M. V. A. Koelman, Simulating microscopic hydrodynamic phenomena with dissipative particle dynamics, Europhys. Lett. 19, 155 (1992). 